\title{
EL EFECTO DEL SALARIO MINIMO SOBRE LAS TRANSICIONES LABORALES EN ARGENTINA. EVIDENCIA A PARTIR DE UN CUASIEXPERIMENTO*
}

\author{
THE EFFECT OF MINIMUM WAGES ON JOB TRANSITIONS IN
} ARGENTINA. EVIDENCE FROM A QUASI-EXPERIMENT

\section{MONICA JIMENEZ**}

Universidad Nacional de Salta

\begin{abstract}
The aim of this paper is to assess the impact of minimum wages on employment quality and the likelihood of transition between jobs of varying quality in both medium and large enterprises and the public sector in Argentina. So far there are no studies with a similar objective in our country. The analysis was developed from data from the Permanent Household Survey using the method of differences in differences. The results indicate that increased minimum wages decreases transitions to quality jobs mainly among informal workers.
\end{abstract}

Keywords: Minimum wage, job transitions, job quality, quasi experiment, Argentina.

JEL Classification: J42, J3, J58, J6, C14.

\section{Resumen}

El objetivo de este artículo es evaluar el impacto del salario mínimo sobre la calidad del empleo y sobre la probabilidad de transición entre puestos de trabajo de distinta calidad tanto en las medianas y grandes empresas como en el sector público de Argentina. Hasta el momento no existen estudios con un objetivo similar en nuestro país. El análisis se desarrolla a partir de los datos de la Encuesta Permanente de Hogares utilizando el método

\footnotetext{
* Agradezco los comentarios recibidos de la Dra. Inmaculada Cebrián López, al Dr. Santos Ruesga y al Dr. Enrique López Baso.

** E-mail: monijimen@gmail.com
} 
de diferencias en diferencias. Los resultados indican que el incremento del salario mínimo disminuye las transiciones hacia empleos de calidad principalmente entre los asalariados informales.

Palabras clave: Salario mínimo, transiciones laborales, calidad del empleo, cuasiexperimento, Argentina.

Clasificación JEL: J42, J3, J58, J6, C14.

\section{INTRODUCCION}

El rol que se adjudica al salario mínimo al igual que sus efectos sociales y económicos han sido objeto de un amplio debate e investigación (Casanova et al., 2015). Los estudios pioneros evaluaron el impacto del SM sobre los salarios y la desigualdad (Card, 1992; Card y Krueger, 1994; Manning y Machin, 1996; entre otros). A partir de ellos se desarrolló una extensa literatura principalmente en los países desarrollados. Gran parte de ellos evaluaron las predicciones teóricas del impacto del SM sobre los niveles de empleo. Así, los modelos neoclásicos de mercado de trabajo predicen una reducción del empleo (Card y Krueger, 1994; González Güemes, 1997; Neumark y Wascher, 2000; Groisman, 2013). Algunos estudios que encuentran una relación negativa entre el SM y la demanda de empleo son el de Fang y Lin (2013) para la región este y central de China, Cebrián et al. (2010) para España, Sabia et al. (2012) para Nueva York, Papps (2012) para Turquía y Laporsek (2013) para los países de la Unión Europea. Pero si el mercado de trabajo no es competitivo, los efectos teóricos de un incremento del SM sobre el empleo serán muy diferentes (Cebrián et al., 2010). En modelos teóricos de monopsonio o de salarios de eficiencia se predice la ausencia de efectos contractivos sobre el empleo (Shapiro y Stiglitz, 1984; Manning, 2003). Entre los diversos estudios recientes a nivel internacional que estiman efectos positivos del SM sobre el empleo pero de una magnitud pequeña se encuentran el de Blažević (2012) y el de Hirsch et al. (2016). En tanto que otros no observan efectos del SM sobre el empleo como Dube et al. (2010) y Addison et al. (2012) en Estados Unidos así como Allegretto et al. (2011), Higuchi (2013) y Dinkelman y Ranchhod (2012) y Bhorat et al. (2013) en Sudáfrica.

La magnitud de los efectos del SM sobre los ingresos, el empleo, y en general, sobre otras variables económicas y sociales, dependerá en gran medida del cumplimiento del SM y, este último, de su nivel. Por esto es necesario considerar el contexto económico y la situación del mercado de trabajo. Casanova et al. (2015) señalan que fuertes aumentos reales del SM en períodos de desaceleración económica pueden ser difíciles de absorber por las empresas y llevar a un aumento en el incumplimiento. En cambio, en contextos de crecimiento económico y de caída en el desempleo los incrementos del SM podrían implementarse sin ocasionar impactos negativos. 
Asimismo, el nivel de cumplimiento del SM también está relacionado con la heterogeneidad estructural de la economía de un país, esto es, la coexistencia de unidades económicas y actividades con marcadas diferencias en cuanto a su desempeño económico. Esta heterogeneidad puede trasladarse a la estructura de remuneraciones de las distintas regiones geográficas. En estos casos, un aumento del SM a nivel nacional podría introducir rigideces en la contratación del empleo formal en aquellas regiones con menos desarrollo y al mismo tiempo estimular el incumplimiento de esta institución laboral. Por tanto, el nivel del SM puede producir diferentes efectos en la composición del empleo a nivel regional y probablemente constituya un piso salarial muy importante en aquellas regiones con alto grado de incumplimiento. En relación con este punto, Saget (2001) señala que el incumplimiento del SM es mayor cuando la relación entre el SM y el salario promedio de la economía es más alta. Se considera que este es un indicador que refleja las condiciones generales de productividad del trabajo así como la capacidad de pago de los empleadores en cada país. Además, Boeri (2009) encuentra que la relación entre el salario mínimo y el salario promedio es mayor cuando el primero se fija en un esquema de negociación tripartita en comparación a cuando el mismo es fijado por el gobierno (Casanova et al., 2015). Otros factores que afectan el nivel de cumplimiento del SM son los institucionales, en particular, aquellos asociados con la ineficacia del sistema de inspección, la falta de divulgación de la información acerca de este, la escasez de inspectores laborales, la poca repercusión de las sanciones, la fragilidad de los sindicatos y de la negociación colectiva (Belser y Sobeck, 2012; Rani et al., 2013; Marinakis y Bueno, 2014; Casanova et al., 2015).

Pero más allá de los aspectos institucionales, las características del empleo son muy relevantes para el alcance y cumplimiento del SM. Al analizar la cobertura jurídica del SM en los países en desarrollo debe tenerse en cuenta el peso que tiene en el empleo total el trabajo informal o no registrado en el sistema de seguridad social. Este tipo de puestos reduce el potencial de las políticas de SM como instrumentos de redistribución de los recursos a favor de todos los trabajadores de baja remuneración. Esto último añade otros interrogantes, sobre todo en países con mercados de trabajos segmentados y de elevada informalidad laboral como Argentina. En primer lugar, resulta relevante averiguar si el SM beneficia a todos los trabajadores o a un conjunto de ellos, ya que hay grandes sectores que se ubican por fuera de toda la cobertura social y por esta razón, de la cobertura del SM. En Argentina, los trabajadores efectivamente alcanzados por esta normativa corresponden al conjunto de los empleados formales. De esta forma, el porcentaje de empleo formal podría estar significando un techo para la tasa de cumplimiento del SM (Casanova et al., 2015). Un incremento en el SM, lejos de beneficiar a cierto grupo de trabajadores, principalmente a los que se encuentran en la parte más baja de la distribución salarial (como los empleados informales), podría perjudicarlos, si se considera que esta institución laboral supone una restricción importante que impone un valor mínimo a una parte relevante de los costes laborales, esto es, al salario. Si el SM eleva demasiado el salario y, con ello, todos los costes laborales que estén calculados en función de este, los empleadores 
podrían impulsar la creación de empleo de baja calidad con el objetivo de evadir su cumplimiento reduciendo la proporción de puestos de trabajo de calidad. En este documento se entiende que la calidad del empleo ${ }^{1}$ hace referencia a un conjunto de características del puesto de trabajo referentes no solo a la protección social sino también a los derechos laborales, a las oportunidades de empleo y al diálogo social (OIT, 2002).

Por otra parte, algunos estudios sostienen que el SM podría crear ciertas rigidices en el mercado de trabajo produciendo un cambio en la composición de la calidad del empleo mediante la reducción de los empleos de calidad y el incremento de los puestos de trabajo con déficit de calidad. Al respecto hay distintas hipótesis en la literatura. Según la corriente de los mercados laborales segmentados las intervenciones del gobierno podrían tener impactos diferenciales en el sector formal e informal (Harris y Todaro, 1970; Mazumdar, 1989; Khamis, 2013). El impacto directo del SM en términos de empleo será una disminución de trabajadores en el sector formal y un aumento en el sector informal (Harrison y Leamer, 1997). En efecto, si los trabajadores del sector informal son sustitutos eficaces de los que trabajan en el sector formal, las empresas pueden optar por contratar trabajadores del sector informal a un menor coste (Millea et al., 2017). En ese sentido esta institución laboral puede tener impactos nocivos en la calidad del empleo (Rani et al., 2013; Casanova et al., 2015) produciendo transiciones entre el sector formal y el informal. Las investigaciones más recientes que examinaron los efectos del SM sobre el nivel de empleo diferenciando los puestos según su calidad son menos numerosas. Entre estas se encuentra la de Nguyen (2013), quien analiza el impacto de un aumento del SM sobre el empleo de los trabajadores en el sector formal en Vietnam. El autor concluye que el aumento del SM en 2005 redujo la proporción de los trabajadores en el sector formal. Campoliet et al. (2014) encontraron que los efectos adversos del SM en el empleo son mayores para los trabajadores permanentes que para los temporarios.

Otra alternativa considerada por las empresas ante un incremento del SM, sobre todo cuando los mecanismos para controlar el cumplimiento de la legalidad no funcionan con efectividad, es contratar trabajadores por un salario inferior al SM incluso dentro del sector cubierto. Esto último implica un fraude de parte de las empresas a esta normativa laboral. En este caso el empleo se reduciría en menor cuantía que en el modelo tradicional (Groisman, 2016). Justamente, Garganta y Gasparini (2015) afirman al respecto que son las empresas las que toman las decisiones de registrar

1 Esta definición se corresponde con el concepto de trabajo decente que introdujo la Organización Internacional del Trabajo (OIT). Respecto de los datos que se disponen para llevar a cabo los objetivos de este estudio los empleos de calidad se definen, en términos empíricos, como aquellos puestos que celebran contratos registrados en el sistema de seguridad social o con cotización en el sistema previsional y además tienen contratos a tiempo indefinido (es decir, declaran estar ocupados en un puesto de trabajo sin período de finalización), se encuentran en plena ocupación (es decir, declaran trabajar 48 horas semanales) y reportan satisfacción laboral (declara no estar en la búsqueda de otro empleo o no desea ni busca más horas de trabajo). Para más detalles ver sección 3. 
sus actividades, de pagar impuestos al trabajo y de cumplir con la normativa legal, asumiendo un rol pasivo los trabajadores. De modo que, mientras en algunas empresas un aumento del SM puede provocar un incremento del empleo informal, en otras puede provocar todo lo contrario (Romero, 2006).

También suele afirmarse que, si la actualización de los salarios mínimos responde a una activa participación de los sindicatos ${ }^{2}$, las probabilidades para el incumplimiento de la normativa se estrechan y es esperable que aumenten las transiciones desde la informalidad a la formalidad (Groisman, 2013). Maloney (1999), por su parte, niega ambas posibilidades pues, para este autor, las transiciones desde la formalidad hacia la informalidad no dependen de cambios en las instituciones laborales como el SM sino de las decisiones de los trabajadores en función de los costes y beneficios asociados con un empleo informal. Según este esquema, la introducción de un SM podría no producir transiciones desde el mercado laboral formal al informal, porque los trabajadores deciden el sector de ocupación comparando el coste versus el beneficio de ser un empleado formal considerando variables no asociadas a esta institución laboral.

Los estudios que constituyen los antecedentes más directos de esta investigación son los que examinan empíricamente los impactos del SM sobre las transiciones laborales. En relación con la literatura previa, existe una cantidad significativamente menor de artículos que analizan esta cuestión particular, pues se trata de una temática relativamente incipiente ${ }^{3}$. Entre ellos se destaca el estudio de Dube et al. (2014), que realiza las primeras estimaciones del efecto del SM sobre los flujos de empleo para el mercado laboral de Estados Unidos. Los hallazgos indican que esta institución laboral produce una caída considerable sobre los flujos laborales (tasas de contratación, separación y rotación en el empleo) entre los adolescentes y trabajadores de restaurantes pero no sobre los stocks. En cambio Higuchi (2013) encuentra que el SM produce transiciones desde empleos informales a empleos formales entre los trabajadores del Japón que se encontraban ocupados en empresas que ofrecen entrenamiento y educación. Brochu y Green (2013), por su parte, investigan las diferencias en las tasas de transición del mercado de trabajo de Canadá para regímenes de SM bajo y alto durante 1979-2008. Los autores comprueban que si bien los salarios mínimos más altos dan lugar a tasas de contratación más bajas, las tasas de separación del empleo resultan menores, debido principalmente a la reducción de los despidos.

En América Latina los estudios que analizan los impactos del SM son crecientes pero muchos países de la región carecen aún de evidencia empírica sobre esta relevante cuestión (Lemos 2009; Khamis, 2013). En Argentina, la mayoría de los estudios empíricos analizan los efectos de esta institución laboral sobre la distribución salarial (Fizsbein, 1992; Kristensen y Cunningham, 2006; Khamis, 2008, Keifman y Maurizio,

2 En la mayoría de los países de la región funcionan consejos de salarios o comisiones tripartitas integradas por gobierno, trabajadores y empresarios.

3 Otra línea de investigación incipiente es el análisis de los efectos a largo plazo del SM sobre el empleo o sobre la tasa de crecimiento del empleo. 
2012; Groisman, 2013, 2016; entre otros) y sobre las probabilidades de obtener un empleo (Beccaria, 2004; Szretter, 2005; Marshall, 2006 y Groisman, 2013), pero muy pocas investigaciones evaluaron la incidencia del SM entre los trabajadores del sector informal. Además la evidencia no es concluyente respecto de los efectos del SM sobre el sector informal. Kostzer (2006) encuentra que el SM en un escenario de equilibrios macroeconómicos no solo no afectó la creación de empleo en general, sino que indujo a la creación de puestos de trabajo registrados. En tanto que Beccaria (2004), Marshall (2006) y Groisman (2013) encuentran que las modificaciones del SM no contrajeron la demanda de empleo, ni incentivaron la precariedad laboral. Maloney y Núñez (2003) muestran que los impactos del SM sobre los mercados laborales informales amplifican sus efectos distorsivos. En una investigación reciente para Argentina, Casanova et al. (2015) caracterizan la evolución del cumplimiento del SM durante 1990-2014 considerando su relación con los cambios ocurridos en la calidad del empleo.

Por otra parte, la evolución del SM en Argentina ${ }^{4}$ presenta un escenario propicio para evaluar los cambios en esta política mediante métodos cuasiexperimentales debido a los fuertes contrastes que se observan en su nivel a lo largo de los años del período. Así, durante 1995-2001 el SM permaneció fijo en términos nominales y relativamente estable en términos reales. Pero a partir de junio del 2003 tuvo lugar una revitalización del SM que dio lugar a sucesivos aumentos de su valor nominal hasta la actualidad. No obstante, el incremento más relevante dentro de este período fue el que ocurrió entre enero y septiembre del 2004, pues implicó el primer aumento del SM en términos reales tras un período de marcada estabilidad. Este incremento del SM puede ser utilizado como un experimento natural para estimar el efecto causal del cambio en esta institución laboral sobre un resultado de interés (Khamis, 2013).

En este marco, el objetivo principal de este artículo consiste en evaluar el impacto del cambio en el nivel del salario mínimo (SM) entre el primer y cuarto trimestre de 2004 sobre la tasa de empleo y sobre la probabilidad de transición hacia un puesto de trabajo de calidad entre los asalariados con déficits de calidad tanto en las medianas y grandes empresas como en el sector público (MGEySP). Específicamente, se busca determinar si el incremento registrado en el SM durante ese período redujo la tasa de entrada a los puestos asalariados de calidad y qué vínculo tiene esta reducción con la segmentación laboral presente en las MGEySP según lo demuestra una investigación previa a esta en Jiménez (2016). En consecuencia, se espera que, dentro de este mercado, el efecto del SM sobre las transiciones laborales a empleos de calidad sea negativo. Si esto no es así, cabrían dos posibilidades: i) que los trabajadores afectados por el SM sean relativamente pocos como para que estadísticamente no genere un impacto negativo o ii) que la hipótesis de segmentación laboral en las MGEySP deba ser rechazada.

4 Ver para más detalles, Casanova et al. (2015). 
Por otra parte, se destaca que el mercado de trabajo de las MGEySP tiene un papel predominante en la economía argentina pues concentra la mayor parte de la fuerza laboral y constituye una importante fuente de renta para el país. Además, según Santarcángelo y Schorr (2000), el grupo de las grandes empresas pueden tener un poder oligopólico sobre los distintos sectores en los que actúa y, por tanto, poseen un alto grado de determinación sobre el sendero por el que transita el conjunto de la economía argentina, definiendo en buena medida la situación del mercado laboral. Así también las medianas y grandes empresas suelen verse afectadas por los aspectos del mercado de trabajo que la legislación laboral modifica. El empleo en el sector público, por su parte, suele sostener los niveles del empleo total en distintas fases del ciclo. Al mismo tiempo, el Estado es responsable de fiscalizar e inspeccionar la calidad de los puestos de trabajo, principalmente en las medianas y grandes empresas.

Es interesante destacar, por otra parte, que las cuestiones sobre evaluación de impacto del SM no se han investigado mucho en América Latina. Así pues el estudio aquí presentado pretende contribuir a esta literatura aportando evidencia acerca del efecto del SM sobre las transiciones laborales a los mejores puestos de trabajo en términos de calidad. En este sentido resulta importante mencionar que, hasta el momento, no existen en Argentina estudios comparativos respecto de esta temática que, por un lado, apliquen la misma metodología y que, por otro, implementen este análisis para el mercado de trabajo de las MGEySP.

La estructura de este documento es la siguiente: en la segunda sección se presenta el marco teórico y los antecedentes empíricos de esta investigación; en la tercera se detallan los datos y se realiza una breve descripción de las características generales del SM en Argentina y en la cuarta sección se describe la metodología que se implementará para estimar los efectos del SM sobre la tasa de empleo y las tasas de transiciones laborales y paso seguido se analizan los resultados obtenidos; finalmente, en la última sección se enuncian las principales conclusiones de este estudio.

\section{EL SALARIO MINIMO EN ARGENTINA: NORMATIVA, EVOLUCION Y CUMPLIMIENTO}

Si bien la Constitución Nacional establece que todos los trabajadores tienen derecho a un salario mínimo, esta institución laboral fue introducida oficialmente en 1964 cuando se sancionó la Ley 16.459 con el objetivo de fijar un piso salarial efectivo que les garantice a los trabajadores un nivel de seguridad de ingresos. No obstante, la Ley de Contrato de Trabajo 20.744 (LCT) en 1974 define al SM como la menor remuneración que debe percibir el trabajador mayor a 18 años, sin cargas de familia, en su jornada legal de trabajo (8 horas diarias o 48 horas semanales) sin importar la categoría o actividades que se llevan a cabo, de modo que le asegure alimentación adecuada, vivienda digna, educación, vestuario, asistencia sanitaria, transporte y esparcimientos, vacaciones y previsión. 
El alcance y modalidad de determinación del SM fueron reformados en 1991 por la Ley de Empleo 24.0135. Conforme a esta ley, el SM rige para los asalariados que trabajan 48 horas semanales en el sector privado (excluyendo a los trabajadores del servicio doméstico y del sector agropecuario ${ }^{6}$ ) y para los trabajadores de la Administración Pública Nacional y de los organismos donde el Estado Nacional actúe como empleador (es decir, no se aplica en el sector público provincial ni municipal). Los trabajadores a tiempo parcial tienen derecho a recibir una remuneración que no puede ser inferior a la proporcional que le corresponda a un trabajador a tiempo completo, establecida por ley o convenio colectivo, de la misma categoría o puesto de trabajo. Si la jornada pactada supera esa proporción, el empleador deberá abonar la remuneración correspondiente a un trabajador de jornada completa.

En cuanto a la determinación del SM, el art. 139 de la Ley de Empleo establece que el SM debe ser fijado por el Consejo del Salario, un órgano tripartito compuesto por el Ministerio de Trabajo de la Nación, los empresarios y los trabajadores teniendo en cuenta los datos de la situación socioeconómica, los objetivos del instituto y la razonabilidad de la adecuación entre ambos (Casanova et al., 2015). El ajuste periódico del SM a cargo del Consejo del Salario se realiza en función de las variaciones del coste de vida medido por el índice oficial y se instituye a nivel federal, pues no existen salarios mínimos a nivel de provincias. Sin embargo, a fines de 1993 se toma la decisión de fijar y congelar el salario mínimo en \$200 nominales. A ese nivel, el SM permanece relativamente estable en términos reales hasta la crisis económica del 2001 (Gráfico 1). Con la devaluación del peso y el aumento del nivel de precios, el SM se deteriora en 2002, pero a partir de julio de 2003 se verifica una intensa política de actualización de su valor nominal. No obstante, aunque el SM se modifica en forma reiterada entre 2002 y 2014, el primer incremento en el SM nominal que conduce a que el SM real creciera y recuperara su valor (después de la crisis del 2001-2002) ocurre entre enero y septiembre del $2004^{7}$ (Khamis, 2013). Durante este período, el SM nominal aumenta de $\$ 350$ a $\$ 450$ (Cuadro A1) produciendo un incremento del 45 por ciento en el SM real respecto del valor que registraba en el 2001. Además,

5 Esta normativa también modificó la composición del Consejo del Empleo, la Productividad y el Salario Mínimo, Vital y Móvil, sus funciones y dinámica operativa. El Consejo está integrado por 16 representantes de los empleadores y 16 de los trabajadores, designados por el Poder Ejecutivo y por un presidente designado por el Ministerio de Trabajo y Seguridad Social, que duran cuatro años en sus funciones. La representación de los trabajadores se integra incluyendo a los trabajadores del sector privado y del sector público de las distintas ramas de actividad. El artículo 137 establece que "las decisiones del Consejo serán tomadas por mayoría de dos tercios. En caso de no lograrse esta al término de dos sesiones, su presidente laudará respecto de los puntos en controversia".

6 De modo que este salario mínimo no se aplica a los trabajadores del sector rural y a los trabajadores domésticos; éstos tienen una normativa propia (aunque la nueva Ley de Trabajo Agrario determina que la remuneración mínima de los trabajadores agrarios no puede ser inferior al Salario Mínimo Vital y Móvil).

7 Este incremento en el SM es el que se considerará en el análisis del efecto del SM sobre las transiciones laborales en las MGEySP. 


\section{GRAFICO 1}

EVOLUCION DEL SALARIO MINIMO EN TERMINOS NOMINALES Y REALES, 1995-2014

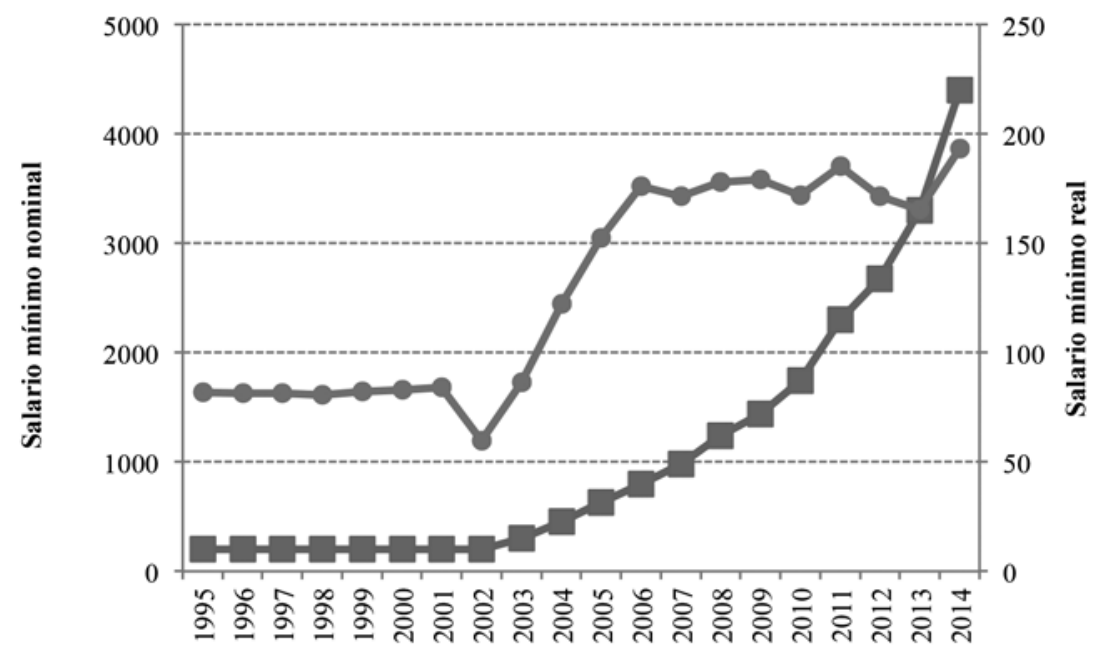

$\longrightarrow$ SM nominal $\longrightarrow$ SM real

Nota: Para expresar el salario mínimo real en moneda constante se utilizó el índice de precios al consumidor elaborado por el INDEC (hasta 2006) y los índices de precios de un conjunto de provincias (desde 2007), continuando una serie de índices de precios provinciales iniciada por el Centro de Estudios para el Desarrollo Argentino (CENDA) para el período 2007-2011.

Fuente: Elaborado a partir de los microdatos de la EPH.

se observa que el aumento del SM en términos nominales entre junio del 2003 y septiembre de 2004 está acompañado por un aumento significativo de la proporción de empleados con salario por debajo del mínimo (Cuadro A2). Asimismo, el aumento del SM en septiembre de 2004 incrementa sustancialmente la relación entre el SM y la remuneración promedio (el índice de Kaitz) tanto entre los AF como entre los AI de las MGEySP (Cuadro A3). Por ello, se espera que este cambio en el SM tenga algún efecto relevante en el mercado de trabajo del sector bajo análisis. Sin embargo, como la actualización del SM se produjo en un contexto económico globalmente favorable que se tradujo en aumentos del empleo y en reducciones del desempleo, la desigualdad y la informalidad laboral, podría esperarse lo contrario. Es decir, en este contexto el efecto negativo del SM sobre la tasa de entrada a puestos de trabajo de calidad podría ser compensado por el efecto positivo del crecimiento económico.

Por otra parte, es relevante considerar la diferente evolución de la tasa de incumplimiento del SM y del índice de Kaitz durante los noventa y los dos mil 
(Cuadro A2 y Cuadro A3). Entre 1995 y 2001 la proporción de asalariados en las MGEySP con un ingreso laboral inferior al SM (ajustado por horas trabajadas) y el índice de Kaitz permanecen relativamente estables. No obstante, durante este período se observan importantes diferencias en el nivel de la tasa de incumplimiento del SM y del índice de Kaitz entre y dentro de los asalariados formales e informales. Mientras el porcentaje de los asalariados formales sin déficit que reciben un salario inferior al mínimo es muy bajo ( 0,2 por ciento) y el índice de Kaitz no supera el 20 por ciento, entre los asalariados formales con déficit ambas medidas son mayores a la de los asalariados formales que no poseen déficit pero menores a las observadas entre los asalariados informales con y sin déficit. Justamente, durante 1995-2001 el porcentaje de incumplimiento del SM (9,2 por ciento en promedio) como el índice de Kaitz ( 38 por ciento en promedio) entre los asalariados informales es significativamente superior, particularmente entre los asalariados informales con déficit, en relación con el observado entre los asalariados formales. Según Casanova et al. (2015) el cumplimiento del SM en cierto modo elevado en los noventa se explica por el nivel relativamente bajo y constante del SM.

Durante la última década se pueden distinguir dos subperíodos. En el primero, 2003-2008, la tasa de incumplimiento del SM entre los asalariados de las MGEySP se incrementa fuertemente del 2,4 por ciento al 14,6 por ciento y el índice de Kaitz crece del 42 al 51 por ciento. El mayor crecimiento de estos indicadores se observa entre los asalariados informales con déficit (casi 47 de la tasa de incumplimiento del SM y 45 puntos porcentuales del índice de Kaitz) y los asalariados formales con déficit (15,5 puntos porcentuales de la tasa de incumplimiento del SM y 29 puntos porcentuales del índice de Kaitz). No obstante, es destacable el elevado porcentaje de asalariados informales con déficit -entre el 37 por ciento y 52 por ciento- cuyos salarios caen por debajo del mínimo durante este período así como el valor del índice de Kaitz para este grupo de asalariados que alcanza hasta cerca del 90 por ciento. Además se aprecia que dentro de este grupo el incremento en la proporción de empleados con salario por debajo del mínimo es mayor que entre los asalariados informales sin déficit (Cuadro A2). De acuerdo con Casanova et al. (2015), el aumento del incumplimiento del SM en la primera etapa responde al deterioro de las condiciones de trabajo debido a que el SM permaneció bajo y casi constante. Además, un porcentaje creciente de los nuevos puestos de trabajo creados eran informales y de baja remuneración. De modo que el nivel del salario mínimo ha sido muy importante en la determinación de los niveles de incumplimiento.

En cambio, en el segundo período, entre 2008-2014, el porcentaje de empleados con salarios por debajo del SM disminuye de casi el 15 por ciento al 8,1 por ciento, mientras el índice de Kaitz se reduce del 53 por ciento al 43 por ciento. La reducción más pronunciada en estas medidas se aprecia entre los asalariados informales con déficit (11 puntos porcentuales). No obstante, el porcentaje de estos trabajadores con salarios por debajo del mínimo y la magnitud del índice de Kaitz continúa siendo elevado, cerca del 37 por ciento y del 72 por ciento, respectivamente. La disminución 
del incumplimiento del SM durante este período, pese al crecimiento del SM, se debe a la mejora en la calidad de empleo que contribuyó a reducir los niveles de incumplimientos que suelen registrarse inmediatamente después de grandes aumentos en el SM (Casanova et al., 2015).

\section{DATOS Y DEFINICIONES}

El análisis empírico de esta investigación está basado en los microdatos provenientes de la Encuesta Permanente de Hogares (EPH) para el período 2004-2005. La EPH es una encuesta urbana llevada a cabo por el Instituto Nacional de Estadísticas y Censos (INDEC) de Argentina en forma conjunta con algunas direcciones provinciales de estadística desde 1974. El objetivo general de la EPH es conocer mediante indicadores un conjunto de dimensiones socioeconómicas básicas de la población como características demográficas, ocupacionales, educativas, del ingreso, de la vivienda y migratorias. Así, la EPH incluye un relevamiento específico sobre el mercado de trabajo conforme a estándares internacionales, con el fin de garantizar cierta comparabilidad. Además de las tasas de actividad, empleo y desempleo, la EPH permite conocer el porcentaje de empleo informal a partir de las declaraciones que realizan los trabajadores asalariados sobre sus contribuciones a la seguridad social (Bertranou et al., 2013). Por esto, constituye la principal fuente de datos sobre variables que caracterizan el funcionamiento del mercado de trabajo. Esta información es recolectada en los principales centros urbanos del país que son capitales de provincias (excepto San Nicolás).

Durante 2004-2005 el SM se incrementó desde \$ 350 en enero de 2004 hasta $\$ 450$ en septiembre del mismo año, luego de haber permanecido fijo en \$ 200 durante gran parte de la década de los noventa (Cuadro A1). Este contraste en la evolución del SM es propicio para poder estimar modelos econométricos que permiten evaluar el impacto del SM utilizando la técnica de diferencias en diferencias. De este modo, para evaluar el impacto del aumento del SM nominal sobre las transiciones laborales en el mercado de trabajo de las MGEySP se construyen dos paneles semestrales ${ }^{8}$.

Aunque la EPH no recolecta información longitudinal es posible construir datos de este tipo debido al esquema de rotación ${ }^{9}$ de esta encuesta. El primer panel corresponde al período previo al incremento observado en el SM nominal, lo que permite hacer un seguimiento de los individuos entrevistados durante el primer y el segundo trimestre

8 Tratándose de paneles cortos el problema de desgaste (attrition) no resulta importante en este caso pues no supera el 10 por ciento.

9 El esquema de rotación de la EPH permite la construcción de paneles cortos de datos. Este esquema tiene la siguiente estructura. Los hogares son incluidos en la encuesta durante dos trimestres consecutivos, luego se retiran temporalmente durante los dos trimestres siguientes y, finalmente, se incorporan a la muestra durante dos trimestres adicionales sucesivos. De esta forma, el solapamiento existente en los paneles confeccionados entre trimestres contiguos y entre idénticos trimestres correspondientes a años inmediatamente consecutivos es de un 50 por ciento de la muestra seleccionada en cada período. 
del 2004. El segundo panel es posterior al mes en que se produce el aumento en el SM y une las observaciones del cuarto trimestre de 2004 y el primer trimestre de 2005.

Esta forma de construir los paneles admite obtener un número aceptable de observaciones. Cada panel cuenta con más de 7.000 observaciones para los asalariados empleados en las MGEySP.

Como la EPH siguió un esquema de incorporación progresiva de áreas urbanas, el período de análisis considerado en este estudio permite seguir a 29 centros urbanos de más de 100 mil habitantes que representan al 71 por ciento de la población urbana de Argentina y al 62 por ciento de la población total del país.

Debido a la normativa vigente con relación a la cobertura jurídica del SM y considerando la población en edad de trabajar, el universo de análisis de este estudio está compuesto por los ocupados mayores de 18 de años y menores de 65 años. Esta restricción se realiza porque la dinámica laboral para individuos menores a 18 y mayores a 65 años puede tener interpretaciones muy diferentes que la del resto de la población (Khamis, 2013).

Los datos acerca de los salarios mínimos se obtuvieron del Ministerio de Trabajo, Empleo y Seguridad Social.

A partir de la información provista por la EPH se clasifican a los asalariados que trabajan en las MGEySP en cuatro categorías laborales que fueron definidas considerando las combinaciones posibles entre la condición de (in)formalidad laboral de los trabajadores y la presencia de déficit en otros atributos que definen la calidad del empleo (satisfacción laboral, estabilidad en el empleo y extensión de la jornada de trabajo: sobreocupación o trabajo a tiempo parcial involuntario). Así se distingue entre:

i) Asalariados formales con déficits en otros atributos de la calidad del empleo (AFCD): empleados que declaran tener déficit en otra dimensión de la calidad del empleo, excluyendo a la referente a la seguridad social. Se trata de asalariados que celebran contratos laborales registrados en el sistema de seguridad social o que poseen cotización en el sistema previsional pero declaran encontrarse en algunas de las siguientes situaciones. Están ocupados en un puesto de trabajo inestable (que tiene un período de finalización), son empleados a tiempo parcial en forma involuntaria (trabajan menos de 32 horas semanales y desean trabajar más) o están sobreocupados (trabajan más de 48 horas semanales) o bien declaran estar insatisfechos con su empleo (están en la búsqueda de otra ocupación o desean o buscan más horas de trabajo).

ii) Asalariados informales con déficits en otros atributos de la calidad del empleo (AICD): empleados que declaran tener déficit en más de un atributo de la calidad del empleo. Se trata de asalariados que no celebran contratos laborales registrados en el sistema de seguridad social o no poseen cotización en el sistema previsional y además declaran estar ocupados en un puesto de trabajo con período de finalización, encontrarse en empleos a tiempo parcial en forma involuntaria (trabajan menos de 32 horas semanales y desean trabajar más) o sobreocupados (trabajan más de 48 
horas semanales) o bien insatisfechos con sus empleos (están en la búsqueda de otro empleo o desean o buscan más horas de trabajo). Este grupo de trabajadores ocupan los peores puestos de trabajo en términos de calidad pues presentan déficits en todos los atributos de la calidad del empleo considerados.

iii) Asalariados informales sin otro déficit en la calidad del empleo (AISD): asalariados que declaran tener déficit solo en una dimensión de la calidad del empleo: en este caso, la referente a la seguridad social. Es decir, se trata de asalariados que no tienen contratos laborales registrados en el sistema de seguridad social o cotización al sistema previsional pero declaran estar ocupados a tiempo indefinido (es decir, sin período de finalización), en trabajos de plena ocupación (trabajan 48 horas semanales) o que tienen satisfacción laboral (no se encuentran en la búsqueda de otro empleo o no desean ni buscan más horas de trabajo).

iv) Asalariados formales sin ningún déficit en la calidad del empleo (AFSD) o asalariados con puestos de trabajo de calidad. Son empleados que declaran celebrar contratos registrados en el sistema de seguridad social o con cotización en el sistema previsional y además tienen contratos a tiempo indefinido (es decir, declaran estar ocupados en un puesto de trabajo sin período de finalización), se encuentran en plena ocupación (es decir, declaran trabajar 48 horas semanales) y reportan satisfacción laboral (declaran no estar en la búsqueda de otro empleo o no desean ni buscan más horas de trabajo). Este grupo de trabajadores ocupan los mejores puestos de trabajo en términos de calidad pues no presentan ningún déficit en la calidad del empleo.

\section{EVALUACION DE LAS HIPOTESIS DE ESTUDIO}

En esta sección se presenta el método empírico utilizado para examinar los efectos del SM sobre la tasa de empleo y las transiciones laborales en las MGEySP y a continuación se analizan los resultados obtenidos de su estimación.

\subsection{Estimación del efecto del SM sobre la tasa de empleo y las transiciones laborales}

En esta sección se describe el método econométrico utilizado para estimar el efecto causal del cambio registrado en el SM en septiembre de 2004 sobre la tasa de empleo y la tasa de entrada a los puestos asalariados formales sin déficit (AFSD) en las MGEySP. Para ello se utiliza el método de diferencias en diferencias (DD) que consiste en comparar las discrepancias en esa tasa de entrada entre un grupo de tratamiento y de control antes y después de la ejecución de la política (Card, 1992; Card y Krueger, 1994). Esta metodología evita varios de los problemas de endogeneidad que surgen al contrastar individuos heterogéneos (Bertrand et al., 2004; Garganta y Gasparini, 2015). 
La implementación de este método requiere, por tanto, la asignación de los individuos de la muestra a dos grupos: uno, formado por los individuos que han sido expuestos a los efectos de un tratamiento, en este caso, el incremento del SM (grupo de tratados) $y$, el otro, constituido por individuos que se han mantenido ajenos al tratamiento (grupo de control).

Para definir el grupo de tratamiento y de control se sigue la estrategia empírica propuesta inicialmente por Stewart (2002) y seguida por Khamis (2013). Esta estrategia consiste en utilizar un cuasiexperimento considerando que los cambios registrados en el SM pudieron tener un impacto diferencial entre las 29 áreas urbanas que incluye la EPH en Argentina. Este impacto diferencial está determinado por la proporción inicialmente diferente de trabajadores con bajos salarios que existe en cada ciudad del país. Para esto se utilizan dos medidas: la tasa de incumplimiento y el índice de Kaitz.

La tasa de incumplimiento se define como el porcentaje del total de los asalariados ${ }^{10}$ (sin distinguir entre formales e informales) que, en cada ciudad, reciben un ingreso laboral horario inferior al nuevo SM por hora. Este SM horario se computó dividiendo el SM mensual por las 192 horas semanales que surgen de considerar la jornada laboral legal de 48 horas semanales ${ }^{11}$. Si se utilizan los salarios por mes y se confrontan con el SM mensual, se podría sobrestimar el incumplimiento entre quienes trabajan jornadas parciales y subestimarlo entre los trabajadores sobreocupados. La tasa de incumplimiento computada de ese modo es utilizada para definir a los grupos de alto y de bajo impacto. Los puntos de corte para la clasificación son determinados, observando la distribución de la tasa de incumplimiento entre las ciudades. Así, conforme con la tasa de incumplimiento computada, es posible distinguir entre un grupo de áreas donde se espera que el incremento del SM tenga un alto impacto, ya que en ellas existe una proporción elevada de empleados con remuneraciones debajo del SM y otro grupo de áreas locales donde se espera un bajo impacto del SM por el bajo porcentaje de empleados con salarios menores al SM. El grupo de áreas urbanas de alto impacto es el grupo de tratamiento y el grupo de ciudades de bajo impacto será el grupo de control. De este modo, los empleados que se encuentran ocupados en las MGEySP se asignan al área urbana correspondiente al grupo de alto y de bajo impacto según la proporción inicial de asalariados que se encuentran por debajo del nuevo SM en su área urbana de residencia. El alcance diferencial del SM entre las 29 ciudades de Argentina es la fuente de variación exógena en esta estrategia de estimación (Khamis, 2013).

10 Se excluye del conjunto de los asalariados a los trabajadores domésticos, porque este grupo tiene su propia normativa referente al SM.

11 En las estimaciones relacionadas con la tasa de incumplimiento se excluyeron del análisis a los ocupados con ingresos laborales iguales a cero y que declaran trabajar más de 84 horas semanales teniendo en cuenta la normativa vigente del SM. 
Como se observa en el Cuadro A4, las tasas de incumplimiento son más elevadas (superiores al 30 por ciento de los asalariados) en la mayoría de las áreas urbanas del país. Mientras que en varias ciudades del centro y del sur del país no superan el 25 por ciento. Esta variabilidad geográfica en el alcance del SM indica que su impacto es probablemente menor en las ciudades del sur y del centro del país que en el resto de las localidades consideradas. Asimismo en el Gráfico 2 se observa la variación geográfica de la tasa de incumplimiento que, del mismo modo que en el Cuadro A4, sugiere un potencial impacto diferencial del incremento del SM por área urbana de Argentina. Las ciudades con una tasa de incumplimiento menor al 29,4 por ciento se clasifican como el grupo de bajo impacto y las que registran una tasa de incumplimiento por encima de ese valor son clasificadas como el grupo de alto impacto. Este valor corresponde al percentil 75 de la distribución de la tasa de incumplimiento del SM entre las áreas urbanas (Cuadro A4). Es importante aclarar que las estimaciones del impacto el SM sobre las transiciones laborales se realizan considerando distintos puntos de corte.

GRAFICO 2

TASA DE INCUMPLIMIENTO DEL SM POR AREA URBANA

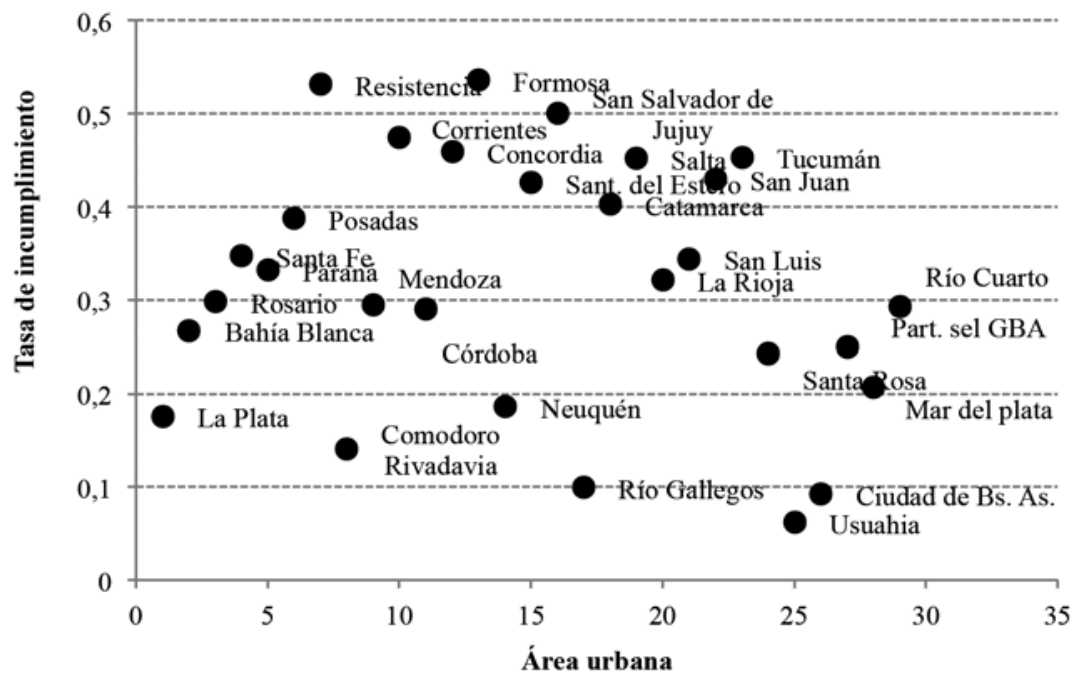

Nota: Las tasas de incumplimiento se computan comparando el ingreso laboral horario de las observaciones incluidas en el primer panel (es decir, entre el primer y segundo trimestre del 2004) con el nuevo SM horario vigente a partir del cuarto trimestre del 2004. Para más detalles ver Cuadro A4.

Fuente: Elaborado a partir de los microdatos de la EPH. 
La segunda variable que se utiliza para evaluar el impacto del SM y clasificar a los asalariados en los grupos de tratamiento y control es el índice de Kaitz (1970) medido como la ratio entre el nuevo SM horario y el salario promedio horario de cada área urbana. El índice de Kaitz computado de este modo muestra la proporción que representa el nuevo SM en relación con el salario medio vigente en cada área urbana. Así, pues, esta medida otorga información acerca de la importancia que tiene el coste salarial de los trabajadores que reciben el SM en relación con la masa salarial global de la empresa. Por lo general se observa que el efecto del SM sobre el empleo tiende a ser mayor cuanto más importante es el número de trabajadores contratados que reciben este salario (Cebrián et al., 2010).

Nuevamente, los puntos de corte para la clasificación de cada grupo son determinados observando la distribución del índice de Kaitz entre las 29 ciudades de Argentina incluidas en la EPH. En el Gráfico 3 se observa la variación geográfica así como el potencial impacto diferencial de este índice por área urbana. En este caso, las ciudades con un índice de Kaitz superior al 60,4 por ciento se clasifican en el grupo de alto impacto y las que poseen un índice por debajo de ese valor constituyen el grupo

\section{GRAFICO 3}

INDICE DE KAITZ POR AREA URBANA

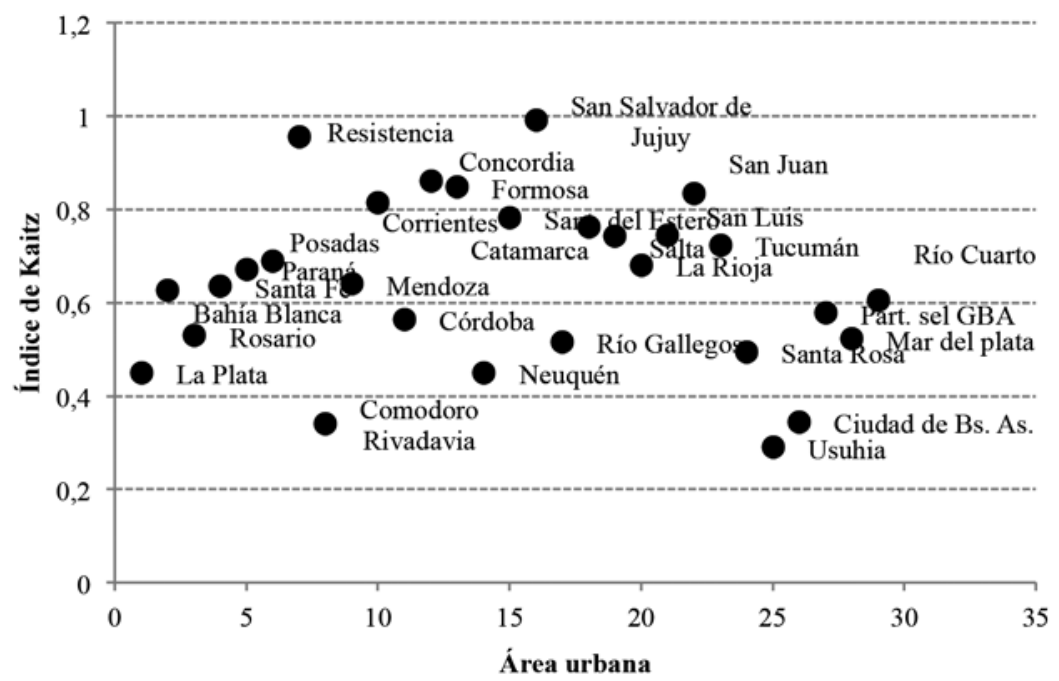

Nota: El índice de Kaitz se computa como la ratio entre el ingreso laboral horario de las observaciones incluidas en el primer panel (es decir, entre el primer y segundo trimestre del 2004) con el nuevo SM horario vigente a partir del cuarto trimestre del 2004. Para más detalles ver Cuadro A4.

Fuente: Elaborado a partir de los microdatos de la EPH. 
de bajo impacto. Este valor corresponde al percentil 75 de la distribución del índice de Kaitz (Cuadro A4). Es admisible aclarar que las estimaciones del impacto el SM sobre las transiciones laborales se realizan considerando distintos puntos de corte.

Asimismo, en el Cuadro A4 se observa una variación geográfica de los valores de este índice similar a la tasa de incumplimiento. Así, mientras la mayoría de las ciudades del centro y del sur del país presentan niveles relativamente menores del índice de Kaitz, el resto de las ciudades muestran los valores más elevados de este indicador. Sin embargo, las áreas urbanas que forman parte del grupo de control y de tratamiento según los valores de esta variable, no son exactamente las mismas que surgen cuando se considera la tasa de incumplimiento. Esto permitirá evaluar si los resultados obtenidos cambian al modificar las ciudades incluidas en el grupo de tratamiento y de control.

Una vez identificados los grupos de tratamiento y de control, para estimar el efecto del incremento del SM sobre la tasa de empleo y la tasa de transición laboral a empleos de calidad dentro de las MGEySP a partir del método DD se estima un modelo no lineal. Debido a que la variable dependiente es una variable binaria que representa probabilidad de encontrarse empleado en un segmento laboral o de transitar desde un puesto con déficit (ya sea un puesto AFCD, AICD o AISD) a otro sin ningún déficit (es decir, los empleos AFSD), el impacto del SM en esta tasa se puede obtener mediante la estimación del siguiente modelo Probit como se explica a continuación:

$$
P\left(T_{i a t}\right)=\Phi\left(\alpha+\beta \text { Post }_{t}+\beta D_{a}+\text { PPost }_{t} D_{a}+\delta X_{\text {iat }}+\gamma Z_{a t}\right)
$$

Donde $P\left(T_{i a t}\right)$ es el resultado que interesa evaluar, en este caso, la probabilidad de que un asalariado se encuentre ocupado en un determinado segmento laboral o transite hacia un puesto de trabajo formal sin ningún déficit (AFSD) durante el período que abarca el panel, ya que se encontraba ocupado inicialmente como empleado en un puesto de trabajo con déficit de calidad de empleo (ya sea porque es un AISD, un AICD o un AFCD). El vector de variables $X$ incluye un conjunto de controles individuales que capturan características demográficas y laborales de los trabajadores para ajustar por diferencias observables entre los individuos que forman parte del grupo de tratamiento como de control que podrían sesgar las estimaciones del coeficiente de interés. Estos controles incluyen variables de género, edad, edad al cuadrado, años de educación, estado civil, la asistencia a un establecimiento educativo, la presencia de un jefe de hogar, dummies de rama de actividad, de calificación de la tarea, de tamaño de la firma y de antigüedad en la ocupación. Asimismo, $Z$ incluye efectos fijos por ciudad como el PBG, el lugar de nacimiento, la tasa de desempleo por área urbana y dummies que identifican a cada ciudad.

La variable $D_{a}$ toma el valor 1 si el i-ésimo individuo reside en el área $a$ que pertenece al grupo de alto impacto (o grupo de tratamiento) y 0 si pertenece al área $a$ de bajo impacto (o grupo de control). La variable Post $_{t}$ distingue las observaciones 
del período posterior al incremento del $\mathrm{SM}^{12}$ de las observaciones correspondientes al período previo a este incremento ${ }^{13}$.

El término de interacción entre la variable $D_{a}$ y Post $_{t}$, previamente descritas, es una variable dummy que identifica a los individuos del grupo de alto impacto después de la introducción del incremento en el SM. Entonces, $\theta$ es el coeficiente de interés que indica la magnitud y el signo del impacto del SM sobre la tasa de entrada a puestos de trabajo de calidad en las MGEySP. Si las transiciones laborales a empleos de calidad son sensibles a los aumentos del SM, el signo del coeficiente estimado para esta variable resultará negativo. Específicamente, el estimador del parámetro $\hat{\theta}_{D D}$ indica, siempre que la estrategia empírica utilizada sea válida, el verdadero efecto causal del tratamiento sobre el resultado de interés.

De este modo, el impacto del SM es estimado mediante:

$$
\tau_{D D}=\Phi\left(\alpha+\beta_{1}+\beta_{2}+\theta+\delta X_{a i t}+\gamma Z_{a t}\right)-\Phi\left(\alpha+\beta_{1}+\beta_{2}+\delta X_{a i t}+\gamma Z_{a t}\right)
$$

Donde $\Phi($.$) representa una función no lineal estrictamente monotónica, el signo de$ $\theta$ siempre coincidirá con el signo del efecto tratamiento. De manera tal que el efecto tratamiento será, entonces, el impacto incremental en la probabilidad provocado por el coeficiente del término de interacción. Es importante remarcar que siendo $\Phi($.$) una$ función no lineal estrictamente monotónica, se evalúa el impacto del SM sobre las transiciones laborales estimando el efecto parcial promedio (APE).

Athey y Imbens (2002) señalan que en el método de DD subyace el supuesto de que la tendencia temporal registrada por los individuos pertenecientes al grupo de control sirve, a modo de variable proxy, para conocer la evolución que hubieran seguido los individuos del grupo de tratamiento en el caso de que no recibieran el tratamiento. Así, pues, el supuesto de identificación clave para que el estimador de DD sea consistente es que $\theta$ sea 0 en ausencia de tratamiento, es decir, sin el incremento del SM. Esto implica que la tasa promedio de entrada a puestos de trabajo de calidad en las ciudades del grupo de control y de tratamiento debe ser la misma en ausencia de un incremento en el SM (Khamis, 2013). Para verificar esto se estimaron las tendencias en las tasas de entrada a puestos de calidad en las MGEySP para el grupo de control y tratamiento, antes y después del cambio registrado en el SM en septiembre de 2004.

En el Gráfico 4 se puede observar la evolución de estas tasas para cada panel semestral según el individuo que pertenece al grupo de tratamiento o de control.

12 Estas observaciones corresponden a las del segundo panel semestral que surge de la unión de los datos correspondientes al cuarto trimestre del 2004 y el primer trimestre del 2005.

13 Estas observaciones corresponden a las del primer panel semestral que permite seguir a los individuos durante el primer y segundo trimestre de 2004. 


\section{GRAFICO 4}

TENDENCIAS EN LAS TASAS DE ENTRADA A PUESTOS DE CALIDAD PARA EL GRUPO DE TRATAMIENTO Y DE CONTROL ANTES Y DESPUES DEL INCREMENTO EN EL SM

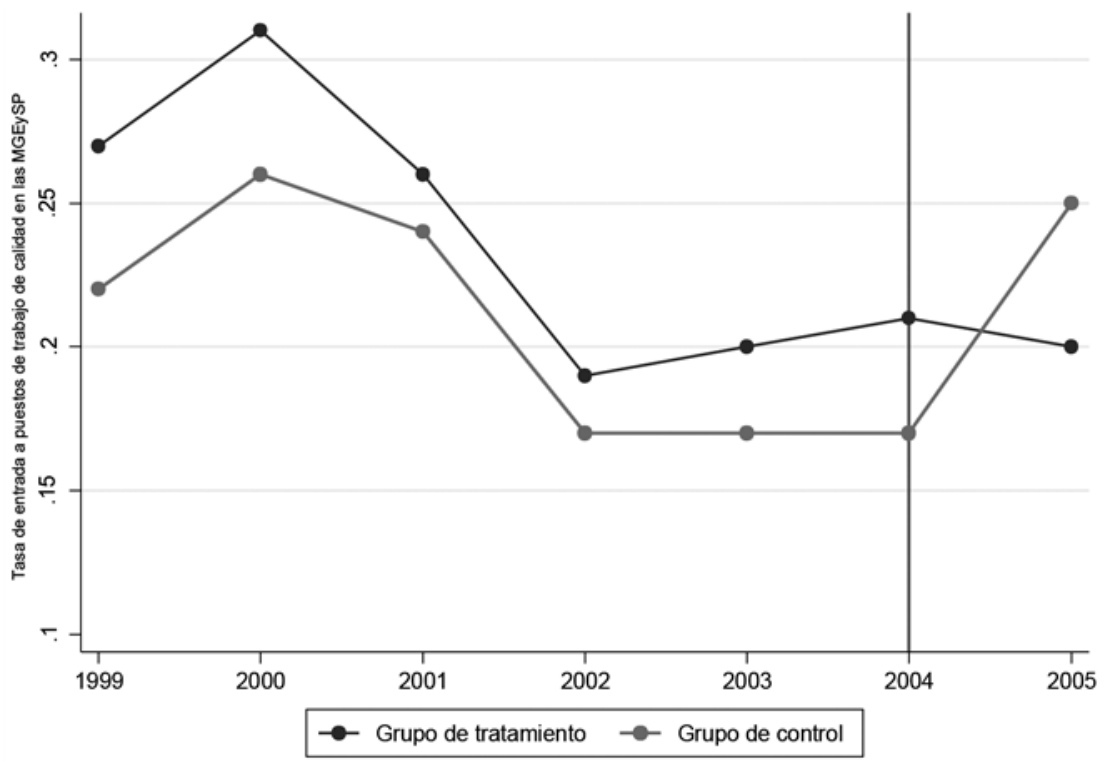

Nota: Las tasas de transición fueron estimadas utilizando los datos de panel correspondientes a la onda mayo y octubre para el período 1999-2002 y al segundo semestre de cada año para el período 2003-2005.

Fuente: Elaborado a partir de los microdatos de la EPH.

Desde 1999 al 2002 se observan tendencias similares en la tasa de entrada a puestos de trabajo de calidad entre un grupo y otro. En cambio, después del incremento modesto del SM nominal en julio del 2003 se observa un leve cambio de tendencia en estas tasas. Así, entre los individuos del grupo de tratamiento se aprecia un suave aumento en la tasa de transición, mientras que en el grupo de control esta tasa permanece estable. Asimismo, después del aumento del SM en septiembre de 2004, las tendencias en las tasas de transición hacia puestos de trabajo de calidad divergen manifiestamente entre el grupo de tratamiento y control. Mientras la proporción de asalariados que accedieron a estos puestos crece fuertemente en el grupo de control, esa proporción presenta un leve descenso entre los trabajadores del grupo de tratamiento.

Asimismo, la correcta estimación del efecto causal del SM requiere la inexistencia de otro evento diferente al cambio en el SM que genere un impacto diferencial entre el grupo de tratamiento y de control sobre el resultado de interés. De hecho, este supuesto parece cumplirse en el caso de este artículo porque en el tercer trimestre 
del 2004, que separa el primer período de observación del segundo período, no se implementaron medidas de política social, laboral o económica, más allá del cambio en el SM con potenciales efectos diferenciales entre los empleados de las MGEySP pertenecientes al grupo de tratamiento y los clasificados en el grupo de control. No obstante, existen otros factores que podrían influir en los resultados y esto a su vez haría dudosa la identificación del efecto causal de un cambio en el salario mínimo en las tasas de transición laboral. Para tener en cuenta estos problemas potenciales en el modelo estimado se incluyeron variables referidas a la antigüedad en la ocupación, la calificación de la tarea, al sector de la industria y al tamaño de la empresa en la que se encuentran empleados los individuos. Se incluyeron también controles locales como la tasa de desempleo y el nivel del Producto Bruto Geográfico (PBG) ${ }^{14}$ de cada provincia y controles a nivel individual, como la edad, el género, la educación, el estado civil, la presencia de un jefe en el hogar y la asistencia a un establecimiento educativo.

\subsection{Resultados del análisis de la hipótesis de estudio}

En esta sección se analiza el impacto del SM sobre la tasa de empleo en primer lugar y luego sobre la tasa de entrada a empleos de calidad en las MGEySP. Con este fin se estiman distintas especificaciones del modelo econométrico (1) descrito en la sección anterior. El resultado de interés en este caso es el efecto marginal del término de interacción del modelo Probit (1) definido en la ecuación (2) ${ }^{15}$.

Antes de evaluar el efecto del SM sobre las transiciones laborales, se analiza primero si está precedido de un impacto contractivo sobre la tasa de empleo total en las MGEySP ${ }^{16}$. También se analiza el impacto del SM sobre el porcentaje de asalariados que se desempeña en cada uno de los segmentos laborales considerados. Específicamente, si el incremento del SM redujo el porcentaje de asalariados formales con o sin déficit y aumentó el porcentaje de AISD o de AICD. Con este fin se estiman distintas especificaciones del modelo Probit (1) considerando como variable dependiente,

14 El PBG es la medida más importante de la producción de la economía de una provincia o área urbana. Se trata de un indicador estadístico que intenta medir el valor total de los bienes y servicios finales producidos dentro de los límites geográficos de esa economía en un período determinado de tiempo, libre de duplicaciones. Se calcula sumando los valores de mercado de todos los bienes y servicios finales de la economía, mediante un complejo mecanismo de cuantificación. El PBG de una jurisdicción determinada refleja la actividad económica de las unidades productivas residentes en esa jurisdicción, siendo igual a la suma de los valores agregados por dichas unidades productivas. El término geográfico implica que el cálculo se refiere a toda la riqueza creada en el territorio de la jurisdicción correspondiente sin considerar el origen de los factores productivos ni la residencia habitual de sus propietarios que pueden ser extrajurisdiccionales (Dirección de Estadísticas de la provincia de Salta, 2012). La información acerca del PBG por provincia se obtuvo a partir de las Direcciones Provinciales de Estadística de cada provincia.

15 Las estimaciones originales de los diferentes modelos probit están disponibles para quien los requiera

16 Esta tasa de empleo se define como el porcentaje de la población en edad de trabajar (de 18 a 64 años) que se encuentra ocupada en las MGEySP. 
en primer lugar, la probabilidad de estar empleado en una MGEySP y, en segundo lugar, la probabilidad de estar trabajando en cada uno de los cuatro segmentos laborales previamente señalados. Los resultados que se reportan en el Cuadro 1 presentan un impacto negativo pero no significativo del SM sobre la tasa de empleo general así como sobre la tasa de asalariados informales con y sin déficit en las MGEySP. En cambio, se observan efectos positivos del SM sobre la probabilidad de estar empleado en un puesto de asalariados formales con déficit o en uno sin déficit. Estos efectos resultaron estadísticamente significativos en algunas especificaciones del modelo (1).

\section{CUADRO 1}

EFECTO DEL SALARIO MINIMO SOBRE LAS PROBABILIDADES DE EMPLEO EN LAS MGEYSP

\begin{tabular}{|c|c|c|c|c|c|}
\hline \multirow{4}{*}{ Especificaciones del modelo } & \multicolumn{5}{|c|}{ Estimaciones de diferencias en diferencias } \\
\hline & \multicolumn{5}{|c|}{ Tasa de empleo } \\
\hline & Total & AFSD & AFCD & AICD & AISD \\
\hline & \multicolumn{5}{|c|}{ Tasa de incumplimiento } \\
\hline Sin controles & $\begin{array}{c}-0,0039 \\
(0,015)\end{array}$ & $\begin{array}{c}-0,0069 \\
(0,029)\end{array}$ & $\begin{array}{c}-0,0035 \\
(0,024)\end{array}$ & $\begin{array}{l}0,0347 \\
(0,021)\end{array}$ & $\begin{array}{c}0,0334^{*} \\
(0,020)\end{array}$ \\
\hline Con características individuales ${ }^{\mathrm{a}}$ & $\begin{array}{l}-0,0080 \\
(0,018)\end{array}$ & $\begin{array}{c}-0,0029 \\
(0,030)\end{array}$ & $\begin{array}{c}-0,0036 \\
(0,025)\end{array}$ & $\begin{array}{c}0,0293^{*} \\
(0,017)\end{array}$ & $\begin{array}{c}0,0331^{*} \\
(0,018)\end{array}$ \\
\hline $\begin{array}{l}\text { Con características individuales, } \\
\text { laborales y controles de área local } \\
\text { incluyendo dummies por aglomerado }\end{array}$ & - & $-0,0116$ & $-0,0298$ & $0,0337 * *$ & $0,0505 * * *$ \\
\hline \multirow{3}{*}{$\begin{array}{l}\text { Con características individuales y } \\
\text { controles de área local incluyendo } \\
\text { dummies por aglomerado }\end{array}$} & - & $(0,044)$ & $(0,027)$ & $(0,017)$ & $(0,021)$ \\
\hline & $-0,0065$ & $-0,0109$ & $-0,0385$ & $0,0338 *$ & $0,0490 * * *$ \\
\hline & $(0,017)$ & $(0,042)$ & $(0,026)$ & $(0,018)$ & $(0,020)$ \\
\hline
\end{tabular}

Nota: AFSD: Asalariados informales sin ningún déficit de calidad del empleo, AFCD: Asalariados formales con déficits en otros atributos de la calidad del empleo, AISD: Asalariados informales sin déficits en otros atributos de la calidad del empleo y AICD: Asalariados informales con déficits en otros atributos de la calidad del empleo. Los errores estándares robustos estimados por cluster se encuentran entre paréntesis. (a) La especificación del modelo con características individuales incluyen variables como el género, edad, edad al cuadrado, años de educación, estado civil, una variable dummy que indica si el individuo asiste a un establecimiento educativo y otra que indica si es jefe de hogar; (b) la especificación del modelo con características individuales y laborales incluye además de las variables mencionadas en (a), variables dummies por rama de actividad, por tamaño de la firma, por antigüedad en la ocupación y por calificación de la tarea (c) la especificación del modelo con características individuales, laborales y controles a nivel local incluye además de todas las variables mencionadas en el modelo (b), el PBG, el lugar de nacimiento y la tasa de desempleo por área urbana y finalmente, (d) en la especificación más completa del modelo con características individuales, laborales y controles a nivel local se consideran todas las variables mencionadas en el modelo (c) y efectos fijos por área urbana.

Fuente: Elaborado a partir de los microdatos de la EPH. 
Por tanto, las probabilidades de estar empleado en estos segmentos laborales parecen estar afectadas por el aumento del SM.

La ausencia de un efecto significativo sobre la tasa de empleo general en las MGEySP así como sobre las probabilidades de estar ocupado en los dos segmentos laborales de los AF analizados, puede responder al siguiente motivo. El 2004, año en el que se produce el aumento del SM bajo análisis, pertenece a un período de fuerte crecimiento económico en Argentina que se extendió varios años y que pudo resultar favorable para que las MGEySP se expandan, incrementen su competitividad y generen recursos suficientes para hacer frente a los incrementos en el SM. En un contexto como este, el objetivo de maximización de ganancias puede ser obtenido sin modificar los niveles de contratación entre los AF. Por último, los resultados también pueden responder, en parte, a la estrategia empírica implementada. Por esto, se realizaron estimaciones para diferentes definiciones de tasa de empleo ${ }^{17}$ así como para distintos puntos de corte de la tasa de incumplimiento del SM.

Las estimaciones indican que el incremento del SM reduce la probabilidad de entrada a empleos asalariados formales sin déficit (AFSD) en las MGEySP (Cuadro 2).

\section{CUADRO 2}

EFECTO DEL SALARIO MINIMO SOBRE LAS PROBABILIDADES DE TRANSICION EN LAS MGEYSP. GRUPO DE TRATAMIENTO Y DE CONTROL DEFINIDOS A PARTIR DE LA TASA DE INCUMPLIMIENTO DEL SM

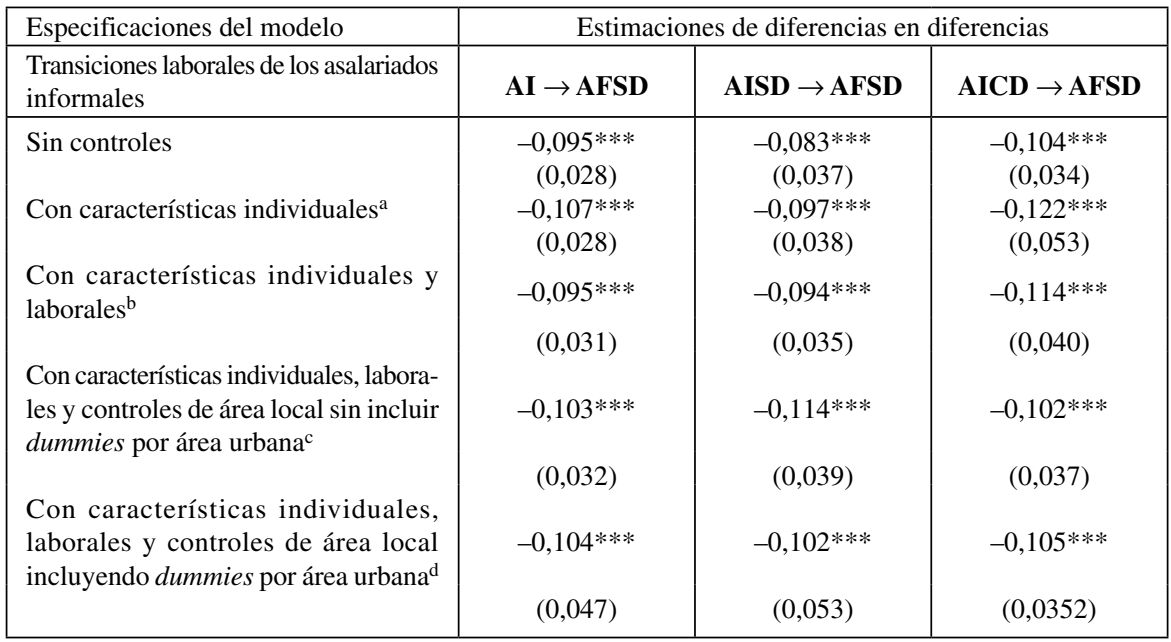

17 Otras medidas utilizadas fueron el porcentaje de la PEA empleado en cada tipo de puesto: AFSD, AFCD, AISD o AICD en las MGEySP. 


\begin{tabular}{|l|c|c|c|}
\hline Especificaciones del modelo & \multicolumn{3}{|c|}{ Estimaciones de diferencias en diferencias } \\
\hline $\begin{array}{l}\text { Transiciones laborales de los asalaria- } \\
\text { dos formales }\end{array}$ & AFCD $\rightarrow$ AFSD & AFCD $\rightarrow$ AI & AFSD $\rightarrow$ AI \\
\hline Sin controles & $-0,025$ & 0,001 & 0,006 \\
& $(0,031)$ & $(0,010)$ & $(0,010)$ \\
Con características individuales & $-0,016$ & 0,001 & 0,005 \\
& $(0,030)$ & $(0,010)$ & $(0,011)$ \\
Con características individuales y & $-0,027$ & 0,008 & 0,012 \\
laborales & $(0,034)$ & $(0,013)$ & $(0,012)$ \\
Con características individuales, labora- & $-0,027$ & 0,009 & 0,011 \\
les y controles de área local sin incluir & & $(0,014)$ & $(0,012)$ \\
dummies por área urbana & & 0,030 & 0,005 \\
Con características individuales, & $-0,009$ & $(0,051)$ & $(0,012)$ \\
$\begin{array}{l}\text { laborales y controles de área local } \\
\text { incluyendo dummies por área urbana }\end{array}$ & $(0,007)$ & & \\
\hline
\end{tabular}

Nota: AFSD: Asalariados informales sin ningún déficit de calidad del empleo, AFCD: Asalariados formales con déficits en otros atributos de la calidad del empleo, AISD: Asalariados informales sin déficits en otros atributos de la calidad del empleo y AICD: Asalariados informales con déficits en otros atributos de la calidad del empleo. Los errores estándares robustos estimados por cluster se encuentran entre paréntesis. (a) La especificación del modelo con características individuales incluyen variables como el género, edad, edad al cuadrado, años de educación, estado civil, una variable dummy que indica si el individuo asiste a un establecimiento educativo y otra que indica si es jefe de hogar; (b) la especificación del modelo con características individuales y laborales incluye además de las variables mencionadas en (a), variables dummies por rama de actividad, por tamaño de la firma, por antigüedad en la ocupación y por calificación de la tarea (c) la especificación del modelo con características individuales, laborales y controles a nivel local incluye además de todas las variables mencionadas en el modelo (b), el PBG, el lugar de nacimiento y la tasa de desempleo por área urbana y finalmente, (d) en la especificación más completa del modelo con características individuales, laborales y controles a nivel local se consideran todas las variables mencionadas en el modelo (c) y efectos fijos por área urbana.

Fuente: Elaborado a partir de los microdatos de la EPH.

En general este resultado es consistente con las predicciones teóricas de un mercado de trabajo segmentado. No obstante, la magnitud del efecto varía con el puesto de trabajo del que provenga el asalariado. En el modelo sin controles, las estimaciones muestran que el cambio en el SM produce una caída de 9,5 puntos porcentuales en la probabilidad de conseguir un empleo de calidad entre los empleados que se encontraban ocupados inicialmente como informales. A medida que se incluyen variables de controles individuales, laborales y a nivel local el efecto del SM sobre esa probabilidad varía levemente desde una magnitud mínima de 9,5 puntos porcentuales hasta un valor máximo de 10,7 puntos porcentuales. Esto sugiere que el resultado de interés es robusto a distintas especificaciones del modelo (1).

Asimismo, se aprecian diferencias marcadas en el impacto estimado entre los asalariados formales con y sin déficit. En general, el efecto negativo del SM en la 
probabilidad de transitar desde un empleo informal hacia un puesto formal sin ningún déficit es mayor entre los AICD que en el caso de los AISD. En la especificación más completa del modelo, mientras el aumento del SM reduce esa probabilidad de transición en 10,2 puntos porcentuales entre los AISD, lo hace hasta en 10,5 puntos porcentuales entre los asalariados informales con déficit.

La evidencia sugiere entonces que el impacto del SM entre los AI es diferente según la calidad del puesto de origen. Esta evidencia es consistente con la existencia de dos segmentos dentro del grupo de empleados informales obtenida en una investigación previa (Jiménez, 2016). Si no existieran estos segmentos, la tasa de entrada a empleos de calidad no debería diferir entre ambos ni tampoco reducirse de forma distinta como resultado de un incremento en el SM.

Tampoco se encuentran entre los asalariados formales con déficit efectos significativos del aumento del SM sobre las probabilidades de transitar a un puesto de trabajo de menor calidad. Si bien los coeficientes estimados son positivos -lo que implica un incremento en la tasa de entrada a empleos informales para este grupo de trabajadores- estos coeficientes no resultan estadísticamente significativos en ningún caso. Del mismo modo, para los empleados que se encuentran ocupados inicialmente como AFSD se halla un efecto positivo pero no significativo como resultado del incremento del SM cuando se estima la tasa de entrada a un puesto asalariado informal o la probabilidad de transitar hacia la desocupación o inactividad.

Ahora bien, si se utiliza el índice de Kaitz para distinguir al grupo de control y tratamiento se obtienen resultados similares (Cuadro 3). El incremento del SM produce un efecto negativo y significativo en la probabilidad de ingresar a un puesto de calidad entre los trabajadores que se encontraban inicialmente ocupados como AICD o como AISD. Además, la magnitud del impacto estimado es similar en ambos grupos de trabajadores y varía de 7,2 puntos porcentuales a 13,7 puntos porcentuales, aproximadamente (Cuadro 3).

\section{CUADRO 3}

EFECTO DEL SALARIO MINIMO SOBRE LAS PROBABILIDADES DE TRANSICION EN LAS MGEYSP. GRUPO DE TRATAMIENTO Y DE CONTROL DEFINIDOS A PARTIR DEL INDICE DE KAITZ

\begin{tabular}{|l|c|c|c|}
\hline Especificaciones del modelo & \multicolumn{3}{|c|}{ Estimaciones de diferencias en diferencias } \\
\hline $\begin{array}{l}\text { Transiciones laborales de los asalariados } \\
\text { informales }\end{array}$ & AI $\rightarrow$ AFSD & AISD $\rightarrow$ AFSD & AICD $\rightarrow$ AFSD \\
\hline Sin controles & $-0,088^{* * *}$ & $-0,072^{*}$ & $-0,081^{* *}$ \\
& $(0,030)$ & $(0,040)$ & $(0,039)$ \\
Con características individuales & $-0,096^{*} * *$ & $-0,081^{* * *}$ & $-0,103^{*}$ \\
& $(0,031)$ & $(0,041)$ & $(0,057)$ \\
Con características individuales y $_{\text {laborales }}^{\mathrm{b}}$ & $-0,096^{* * *}$ & $-0,097^{* * *}$ & $-0,087^{*}$ \\
& $(0,028)$ & $(0,033)$ & $(0,046)$ \\
\hline
\end{tabular}




\begin{tabular}{|c|c|c|c|}
\hline Especificaciones del modelo & \multicolumn{3}{|c|}{ Estimaciones de diferencias en diferencias } \\
\hline $\begin{array}{l}\text { Con características individuales, labora- } \\
\text { les y controles de área local sin incluir } \\
\text { dummies por área urbana }{ }^{\mathrm{c}} \\
\text { Con características individuales, labora- } \\
\text { les y controles de área local incluyendo } \\
\text { dummies por área urbana }{ }^{\mathrm{d}}\end{array}$ & $\begin{array}{c}-0,100 * * * \\
(0,029) \\
-0,109 * * * \\
(0,052)\end{array}$ & $\begin{array}{c}-0,106 * * * \\
(0,036) \\
-0,094 * * * \\
(0,046)\end{array}$ & $\begin{array}{l}-0,08 * \\
(0,043) \\
-0,137 * * \\
(0,060)\end{array}$ \\
\hline $\begin{array}{l}\text { Transiciones laborales de los asalariados } \\
\text { formales }\end{array}$ & AFCD $\rightarrow$ AFSD & $\mathbf{A F C D} \rightarrow \mathbf{A I}$ & $\mathbf{A F S D} \rightarrow \mathbf{A I}$ \\
\hline $\begin{array}{l}\text { Sin controles } \\
\text { Con características individuales }^{\mathrm{a}}\end{array}$ & $\begin{array}{l}-0,030 \\
(0,032) \\
-0,027 \\
(0,031)\end{array}$ & $\begin{array}{c}0,005 \\
(0,012) \\
0,007 \\
(0,013)\end{array}$ & $\begin{array}{c}0,009 \\
(0,012) \\
0,009 \\
(0,012)\end{array}$ \\
\hline $\begin{array}{l}\text { Con características individuales y } \\
\text { laborales }^{\text {b }}\end{array}$ & $\begin{array}{l}-0,053^{*} \\
(0,032)\end{array}$ & $\begin{array}{l}0,007 \\
(0,013)\end{array}$ & $\begin{array}{c}0,014 \\
(0,013)\end{array}$ \\
\hline $\begin{array}{l}\text { Con características individuales, labora- } \\
\text { les y controles de área local sin incluir } \\
\text { dummies por área urbana }{ }^{\mathrm{c}}\end{array}$ & $\begin{array}{l}-0,053^{*} \\
(0,031)\end{array}$ & $\begin{array}{l}0,008 \\
(0,014)\end{array}$ & $\begin{array}{l}0,013 \\
(0,013)\end{array}$ \\
\hline $\begin{array}{l}\text { Con características individuales, labora- } \\
\text { les y controles de área local incluyendo } \\
\text { dummies por área urbana }{ }^{\mathrm{d}}\end{array}$ & $\begin{array}{l}0,005 \\
(0,004)\end{array}$ & $\begin{array}{l}0,006 \\
(0,001)\end{array}$ & $\begin{array}{l}0,001 \\
(0,007)\end{array}$ \\
\hline
\end{tabular}

Nota: AFSD: Asalariados informales sin ningún déficit de calidad del empleo, AFCD: Asalariados formales con déficits en otros atributos de la calidad del empleo, AISD: Asalariados informales sin déficits en otros atributos de la calidad del empleo y AICD: Asalariados informales con déficits en otros atributos de la calidad del empleo. Los errores estándares robustos estimados por cluster se encuentran entre paréntesis. (a) La especificación del modelo con características individuales incluyen variables como el género, edad, edad al cuadrado, años de educación, estado civil, una variable dummy que indica si el individuo asiste a un establecimiento educativo y otra que indica si es jefe de hogar; (b) la especificación del modelo con características individuales y laborales incluye además de las variables mencionadas en (a), variables dummies por rama de actividad, por tamaño de la firma, por antigüedad en la ocupación y por calificación de la tarea (c) la especificación del modelo con características individuales, laborales y controles a nivel local incluye además de todas las variables mencionadas en el modelo (b), el PBG, el lugar de nacimiento y la tasa de desempleo por área urbana y finalmente, (d) en la especificación más completa del modelo con características individuales, laborales y controles a nivel local se consideran todas las variables mencionadas en el modelo (c) y efectos fijos por área urbana.

Fuente: Elaborado a partir de los microdatos de la EPH.

Asimismo, las estimaciones del modelo que incluye características individuales, laborales y controles de área local sin incluir dummies por área urbana indican que entre los asalariados formales con déficit el incremento del SM reduce en 5,3 puntos porcentuales sus probabilidades de ingresar a puestos AFSD. Este resultado difiere del 
obtenido cuando se utiliza la tasa de incumplimiento como variable para identificar al grupo de tratamiento y de control. Sin embargo, como se observa en los resultados del Cuadro 1, entre los AFCD no se aprecia un impacto significativo del SM sobre las probabilidades de transitar a un puesto informal o a salir del mercado de trabajo.

Entre los asalariados formales sin déficit, los diferentes modelos estimados indican que el aumento del SM no produce un efecto significativo sobre la probabilidad de transitar hacia un puesto de peor calidad o de salir del mercado de trabajo. Este resultado coincide del obtenido con la tasa de incumplimiento. Por tanto, el efecto del SM sobre este tipo de transiciones laborales de los AFSD no depende de la medida utilizada para clasificar al grupo de control y de tratamiento.

Por otra parte, como análisis de robustez se utilizan distintos puntos de corte para la tasa de incumplimiento y el índice de Kaitz, con el fin de clasificar a los empleados en el grupo de tratamiento y de control. Los resultados muestran que cuando se establecen puntos de cortes más altos al valor del original las estimaciones no varían demasiado. Sin embargo cuando se consideran puntos de corte menores al original no se obtienen efectos significativos. Esto indica que el salario mínimo (SM) produce un efecto sobre las transiciones laborales cuando el valor de la tasa de incumplimiento del SM o el índice de Kaitz en la ciudad de residencia es mayor o igual al percentil 75 de la distribución de estos indicadores. Es decir, que las modificaciones en el SM generan un impacto solo en las transiciones laborales cuando el porcentaje de asalariados con ingresos laborales inferiores al SM es relativamente elevado. Asimismo, debe tenerse en cuenta que solo se cuenta con información para 29 áreas urbanas del país en la encuesta utilizada, de manera que si el punto de corte se mueve un poco no se dispone de suficientes áreas urbanas en el grupo de control o en el de tratamiento.

Entonces, los resultados más robustos sugieren que el incremento del SM reduce, entre los asalariados informales, las probabilidades de transitar hacia puestos de mayor calidad dentro de las MGEySP. Esto sucede incluso en un contexto económico favorable y dentro de un mercado que se supone altamente visible y sujeto al control fiscal por parte del Estado. Según las predicciones de los modelos teóricos de segmentación laboral esta evidencia sugiere la presencia de segmentos en el mercado de trabajo de las MGEySP.

No obstante, es importante señalar que los efectos negativos de los aumentos del SM no implican bajo ningún punto de vista que deba eliminarse esta institución laboral por varios motivos. La disminución de las tasas de entrada a puestos de trabajo de calidad frente al aumento del SM está relacionada principalmente con la estructura segmentada del mercado de trabajo de las MGEySP. De modo que las políticas públicas deberían dirigirse también a incentivar la creación de mejores puestos de trabajo o a reducir los déficits de calidad del empleo que producen la segmentación de este mercado. Por otro lado, hay evidencia empírica que muestra la existencia de efectos positivos del SM como, por ejemplo, reducciones sobre los niveles de pobreza y de desigualdad, entre otros. 


\section{CONCLUSIONES}

En este artículo se evalúa el impacto del incremento del SM en septiembre de 2004 sobre la tasa de entrada a empleos de calidad en las MGEySP en Argentina. Para ello se utiliza una metodología no experimental de diferencias en diferencias explotando la variación geográfica del alcance del SM (medido a partir de la tasa de incumplimiento y el índice de Kaitz) entre las 29 áreas urbanas cubiertas por la base de datos utilizada (la EPH). Con este fin se construyen dos paneles semestrales correspondientes al 2004, período previo al cambio en el SM, y al 2005, posterior a dicho cambio.

Los resultados obtenidos indican que el incremento del SM produce una disminución significativa en las probabilidades de transitar hacia empleos de calidad principalmente entre aquellos empleados que trabajan como asalariados informales en el período previo. Asimismo, la magnitud del efecto es mayor entre los asalariados informales con déficit de calidad del empleo que entre los que no poseen déficit. En cambio, para los trabajadores ocupados inicialmente como asalariados formales con o sin déficit no se observa un impacto significativo del aumento en el SM sobre sus probabilidades de transitar hacia puestos formales sin ningún déficit.

Según las predicciones de los modelos teóricos de segmentación laboral, un incremento del SM reduce las probabilidades de transitar desde puestos de peor calidad hacia los de mejor calidad. Esto se observa en Argentina, en un contexto económico favorable que parece no compensar el efecto negativo de los cambios en el SM sobre esta transición. Por tanto, si el nivel del SM eleva demasiado el salario, y con ello todos los costes laborales que estén calculados en función de este, los empleadores pueden buscar mecanismos para evadir su cumplimiento. Según Beccaria y Galin (2002), si el SM supera los dos tercios del salario pagado, el mayor coste laboral para las empresas medianas y grandes redundaría en reducciones de la cobertura y en aumentos de informalidad de los asalariados.

Los efectos negativos del SM encontrados no deben ser interpretados a favor de la eliminación de esta institución laboral, pues estos efectos se producen como resultado de la presencia de los fenómenos que segmentan el mercado de trabajo en las MGEySP, tanto de la informalidad laboral como de los déficits en los otros atributos de la calidad del empleo. Por tanto, las políticas públicas, lejos de eliminar el SM, deberían incrementar la entrada a puestos de trabajo de calidad. Finalmente, se recalca que si bien el SM puede causar algunos resultados no deseados en el mercado de trabajo como los que se observan aquí, también produce efectos positivos como disminuciones en los niveles de pobreza y desigualdad de ingresos (Escobar Toledo, 2014; Litwin, 2015; Autor et al., 2016; entre otros). El cumplimiento del SM es un factor esencial para el logro de esos objetivos. Para esto resultan claves las acciones, programas y políticas que favorezcan la formalización del empleo, particularmente en segmentos críticos de alta informalidad, como, por ejemplo, la inspección laboral, la difusión de información sobre el derecho de los trabajadores, entre otros. Asimismo, 
las medidas tendientes a la promoción del empleo registrado y prevención del fraude laboral, como las previstas en la reciente Ley 26.940 del 2014, podrían tener un impacto sobre la tasa de cumplimiento del SM, además de contribuir a disminuir la informalidad del empleo ${ }^{18}$ (Casanova et al., 2015).

\section{BIBLIOGRAFIA}

ADDISON, J. T.; M. L. BLACKBURN y Ch. D. COTTI (2012). "The Effect of Minimum Wages on Labour Market Outcomes: County-Level Estimates from the Restaurant-and-Bar Sector”, British Journal of Industrial Relations, 50(3), pp. 412-435.

ALLEGRETTO, S.; A. DUBE y M. REICH (2011). "Do Minimum Wages Really Reduce Teen Employment? Accounting for Heterogeneity and Selectivity in State Panel Data", A Journal of Economy and Society, 50(2), pp. 205-240.

ATHEY, S. y W. G. IMBENS (2002). "Identification and Inference in Nonlinear difference-in-differences Models". Econometrica, 74(2), pp. 431-497.

BERTRANOU, F.; L. CASANOVA; M. JIMENEZ y M. JIMENEZ (2014). "Informalidad, Calidad del Empleo y Segmentación Laboral”, Revista de Economía Laboral, 11, pp. 24-64.

BECCARIA, L. y P. GALIN (2002). Regulaciones laborales en Argentina. Evaluación y propuestas, Miño y Dávila, Buenos Aires.

BHORAT, H.; R. KANBUR y N. MAYET (2013). "The Impact of Sectorial Minimum Wage Laws on Employment, Wages, and Hours of Work in South Africa", Journal of Labor \& Development, 2(1), pp. 2-27.

BROCHU, P. y D. A. GREEN (2015). “The Impact of Minimum Wages on Labour Market Transitions", The Economic Journal, 123(573), pp. 1203-1235.

CARD, D. (1992). "Using Regional Variation in Wages to Measure the Effects of the Federal Minimum Wage", Industrial and Labor Relations Review, 46(1), pp. 22-37.

CARD, D. y A. B. KRUEGER (1994). "Minimum Wages and Employment: A Case Study of the Fast-Food Industry in New Jersey and Pennsylvania", The American Economic Review, 84(4), pp. 772-793.

CASANOVA, L. y J. ALEJO (2014). "El Rol de la Negociación Colectiva en la Distribución de los Ingresos Laborales. Evidencia Empírica para Argentina en los 2000s", Documento de trabajo No 8, Oficina Internacional del Trabajo, Buenos Aires.

CEBRIAN, I. L.; J. PITACHR; J. RODRIGUEZ y L. TOHARIA (2010). "Análisis de los Efectos del Aumento del Salario Mínimo sobre el Empleo de la Economía Española", Revista de Economía Laboral, 7, pp. 1-38.

DINKELMAN, T. y V. RANCHHOD (2012). "Evidence on the impact of minimum wage laws in an informal sector: Domestic workers in South Africa", Journal of Development Economics, 99(1), pp. 27-45.

DUBE, A.; T. W. LESTER y M. REICH (2010). "Minimum Wage Effects Across State Borders: Estimates Using Contiguous Counties", The Review of Economics and Statistics, 92(4), pp. 945-964.

FIZSBEIN, A. (1992). "Do Workers in the Informal Sector Benefit from Cuts in the Minimum Wage?", Policy Research Working Paper $N^{\circ} 826$.

GARGANTA, L. y L. GASPARINI (2015). "The Impact of a Social Program on Labor Informality: The case of AUH in Argentina", Journal of Development Economics, 115, pp. 99-110.

GONZALEZ GÜEMES, I. (1997). "Los Efectos del Salario Mínimo sobre el Empleo de los Adolescentes, Jóvenes y Mujeres: Evidencia Empírica para el Caso Español”, Cuadernos Económicos de ICE 63, pp. 31-48.

18 Para un análisis más detallado acerca de intervenciones para avanzar en la formalización del empleo en Argentina ver Bertranou y Casanova (2013). 
GROISMAN, F. (2010). "Inestabilidad de Ingresos y Desigualdad Durante la Reciente Fase de Recuperación Económica en Argentina", Estudios del Trabajo 36.

GROISMAN, F. (2013). "Salario Mínimo y Empleo en Argentina”, Revista de economía política de Buenos Aires, 11, pp. 1-40.

GROISMAN, F. (2016). "Una Aproximación a los Efectos Derrame del Salario Mínimo en la Estructura de Remuneraciones de Argentina”, Cuadernos de Economía, 35(68), pp. 457-474.

HARRIS, J. R. y M. P. TODARO (1970). "Migration, Unemployment and Development: A two-sector Analysis", American Economic Review, 60(1), pp. 126-142.

HARRISON, A. y E. LEAMER (1997). "Labor Markets in Developing Countries: an Agenda for Research", Journal of Labor Economics, 15(3), pp. 1-19.

HIGUCHI, Y. (2013). "The Dynamics of Poverty and the Promotion of Transition from non-regular to Regular Employment in Japan: Economic Effects of Minimum Wage Revision and Job Training Support", The Japanese Economic Review, 64(2), pp. 147-200.

HIRSCH, B. (2016). “Dual Labor Markets at Work: The Impact of Employers' Use of Temporary Agency Work on Regular Workers' Job Stability”, Industrial Relations and Labor, 10(26), pp. 1191-1215.

JIMENEZ, M. (2016). "Las Características del Empleo y sus Consecuencias para el Mercado de Trabajo de las Medianas y Grandes Empresas y del Sector Público de Argentina”, Revista Economía (forthcoming).

KAITZ, H. (1970). "Experience of the Past: the National Minimum, Youth at the Unemployment and Minimum Wages", US Bureau of Labour Statistics Bulletin, 1657, pp. 30-54.

KHAMIS, M. (2013). "Does the minimum wage have a higher impact on the informal than on the formal labour market? Evidence from quasi-experiments", Applied Economics, 45(4), pp. 477-495.

KOSTZER, D. (2006). "Argentina: La Recuperación del Salario Mínimo como Herramienta de Política de Ingresos”, en Marinakis, A. y Velasco, J. J. (eds.), ¿Para qué Sirve el Salario Mínimo? Elementos para su Determinación en los Países del Cono Sur. Santiago de Chile: OIT.

KRISTENSEN, N. y W. CUNNINGHAM (2006). "Do Minimum Wages in Latin America and the Caribbean Matter? Evidence from 19 countries”, World Bank Policy Research Working Paper N 3870.

LEMOS, S. (2009). "Minimum Wage Effects in a Developing Country," Labour Economics 16(2), pp. $224-237$.

MALONEY, W. F. (1999). "Does Informality Imply Segmentation in Urban Labor Markets? Evidence from Sectorial Transitions in Mexico", The World Bank Economic Review, 13(2), pp. 275-302.

MALONEY, W. F. y J. NUÑEZ MENDEZ (2004). "Measuring the Impact of Minimum Wages Evidence from Latin America”, en Heckman, J. y Pagés, C. (eds.), Law and Employment: Lessons from Latin America and the Caribbean, Chicago: University of Chicago Press. pp. 109-130.

MANNING, A. y S. MACHIN (1996). "Employment and the Introduction of a Minimum Wage in Britain", Economic Journal, 41(2), pp. 667-676.

MARINAKIS, A. (2006). "Desempolvando el Salario Mínimo: Reflexiones a partir de la Experiencia en el Cono Sur”, en A. Marinakis y Velasco, J. J. (eds.), ¿Para Qué Sirve el Salario Mínimo? Elementos para su determinación en los países del Cono Sur. Buenos Aires: Oficina Internacional del Trabajo.

MARINAKIS, A. (2008). "Evolución de los Salarios en América Latina. 1995-2006”, Organización Internacional del Trabajo.

MARINAKIS, A. y C. BUENO (2014). "Incumplimiento con el Salario Mínimo: ¿Culpa del Nivel o Debilidad Institucional?”, en Marinakis, A. (ed.), Incumplimiento con el Salario Mínimo en América Latina. El Peso de los Factores Económicos e Institucionales. Santiago de Chile: Oficina Internacional del Trabajo.

MARSHALL, A. (2004). "Labour Market Policies and Regulations in Argentina, Brazil and Mexico: Programmes and Impacts", Employment Strategy Paper 2004/13, ILO, Geneva.

MARSHALL, A. (2006). "Salario Mínimo, Mercado de Trabajo y Pobreza. Argentina (2003-2005)", en Informalidad, pobreza y Salario Mínimo. Buenos Aires: Oficina de la Organización Internacional del Trabajo en Argentina.

MAURIZIO, R. (2012). "Labour Informality in Latin America: The Case of Argentina, Chile, Brazil and Peru”, BWPI Working Paper No 165 . Universidad Nacional de General Sarmiento.

MAURIZIO, R. (2014). "El Impacto Distributivo del Salario Mínimo en Argentina, el Brasil, Chile y Uruguay", Serie Políticas Sociales No 194, CEPAL. Santiago de Chile.

MILLEA, M. J.; J. P. REZE; B. SHOUP y J. PITTS (2017). "Minimum Wages in a Segmented Labor Market: Evidence from South Africa", Journal of Labor Research, forthcoming. 
NEUMARK, D. y W. WASCHER (2000). "Minimum Wages and Employment: A Case Study of the Fast-Food Industry in New Jersey and Pennsylvania: Comment", The American Economic Review, 90(5), pp. 1362-1396.

NGUYEN, V. C. (2013). “The Impact of Minimum Wages on Employment of Low-wage Worker, Evidence from Vietnam", Economics of Transition, 21(3), pp. 583-615.

RANI, U.; P.BELSER; M. OELZ y S. RANJBAR (2013). "Cumplimiento y Cobertura del Salario Mínimo en Países en Desarrollo", Revista Internacional del Trabajo, 132(3), pp. 425-457.

ROMERO, P. (2006). "Impacto del Salario Mínimo en la Dinámica de la Formalidad Laboral en Chile". Trabajos de Investigación en Políticas Públicas No 18, Departamento de Economía, Universidad de Chile. SHAPIRO, C. y J. STIGLITZ (1984). "Equilibrium Unemployment as a Worker Discipline Device", American Economic Review, 74(2), pp. 433-44.

STEWART, M. B. (2002). "Estimating the Impact of the Minimum Wage Using Geographical Wage Variation", Oxford Bulletin of Economics and Statistics, 64(5), p. 583-605. 


\section{ANEXO}

\section{A1. CUADROS}

\section{CUADRO A1}

EVOLUCION DEL SALARIO MINIMO. 1993-2015

\begin{tabular}{|c|c|c|}
\hline \multicolumn{2}{|c|}{$\begin{array}{c}\text { Períodos en los que } \\
\text { cambia el valor del salario } \\
\text { mínimo }\end{array}$} & \multirow[t]{2}{*}{$\begin{array}{l}\text { Salario } \\
\text { mínimo } \\
\text { nominal }\end{array}$} \\
\hline Año & Mes & \\
\hline 1993 & Agosto & 200 \\
\hline 2003 & Julio & 250 \\
\hline 2003 & Agosto & 260 \\
\hline 2003 & Septiembre & 270 \\
\hline 2003 & Octubre & 280 \\
\hline 2003 & Noviembre & 290 \\
\hline 2003 & Diciembre & 300 \\
\hline 2004 & Enero & 350 \\
\hline 2004 & Septiembre & 450 \\
\hline 2005 & Mayo & 510 \\
\hline 2005 & Junio & 570 \\
\hline 2005 & Julio & 630 \\
\hline 2006 & Agosto & 760 \\
\hline 2006 & Septiembre & 780 \\
\hline 2006 & Noviembre & 800 \\
\hline 2007 & Agosto & 900 \\
\hline 2007 & Octubre & 960 \\
\hline 2007 & Diciembre & 980 \\
\hline 2008 & Agosto & 1200 \\
\hline 2008 & Diciembre & 1240 \\
\hline 2009 & Agosto & 1400 \\
\hline 2009 & Octubre & 1440 \\
\hline 2010 & Enero & 1500 \\
\hline 2010 & Agosto & 1740 \\
\hline 2011 & Enero & 1840 \\
\hline 2011 & Septiembre & 2300 \\
\hline 2012 & Septiembre & 2670 \\
\hline 2013 & Febrero & 2875 \\
\hline 2013 & Agosto & 3300 \\
\hline 2014 & Enero & 3600 \\
\hline 2014 & Septiembre & 4400 \\
\hline
\end{tabular}

Fuente: Elaboración a partir de los datos del MTEySS. 


\section{CUADRO A2}

TASA DE INCUMPLIMIENTO DEL SALARIO MINIMO. ASALARIADOS DE LAS MGEYSP, 1995-2014

\begin{tabular}{|c|c|c|c|c|c|c|c|}
\hline \multirow{3}{*}{ Años } & \multicolumn{6}{|c|}{ Asalariados } & \multirow{3}{*}{ Todos } \\
\hline & \multicolumn{3}{|c|}{ Formales } & \multicolumn{3}{|c|}{ Informales } & \\
\hline & Todos & Con déficit & Sin déficit & Todos & Con déficit & Sin déficit & \\
\hline 1995 & 0,007 & 0,013 & 0,002 & 0,069 & 0,085 & 0,022 & 0,017 \\
\hline 1996 & 0,007 & 0,014 & 0,001 & 0,073 & 0,094 & 0,013 & 0,018 \\
\hline 1997 & 0,008 & 0,014 & 0,002 & 0,081 & 0,095 & 0,032 & 0,022 \\
\hline 1998 & 0,010 & 0,018 & 0,004 & 0,060 & 0,067 & 0,031 & 0,021 \\
\hline 1999 & 0,007 & 0,012 & 0,002 & 0,070 & 0,081 & 0,030 & 0,019 \\
\hline 2000 & 0,009 & 0,017 & 0,001 & 0,085 & 0,105 & 0,019 & 0,024 \\
\hline 2001 & 0,011 & 0,021 & 0,002 & 0,097 & 0,117 & 0,030 & 0,030 \\
\hline 2002 & 0,011 & 0,021 & 0,002 & 0,098 & 0,109 & 0,059 & 0,029 \\
\hline 2003 & 0,009 & 0,018 & 0,002 & 0,054 & 0,059 & 0,036 & 0,024 \\
\hline 2004 & 0,036 & 0,059 & 0,020 & 0,156 & 0,202 & 0,126 & 0,072 \\
\hline 2005 & 0,075 & 0,140 & 0,033 & 0,502 & 0,521 & 0,259 & 0,204 \\
\hline 2006 & 0,072 & 0,138 & 0,033 & 0,462 & 0,513 & 0,262 & 0,172 \\
\hline 2007 & 0,084 & 0,173 & 0,032 & 0,453 & 0,523 & 0,278 & 0,165 \\
\hline 2008 & 0,083 & 0,156 & 0,041 & 0,440 & 0,489 & 0,367 & 0,146 \\
\hline 2009 & 0,086 & 0,163 & 0,045 & 0,404 & 0,464 & 0,297 & 0,138 \\
\hline 2010 & 0,073 & 0,137 & 0,038 & 0,415 & 0,507 & 0,293 & 0,134 \\
\hline 2011 & 0,063 & 0,124 & 0,031 & 0,373 & 0,456 & 0,235 & 0,113 \\
\hline 2012 & 0,050 & 0,094 & 0,030 & 0,313 & 0,396 & 0,205 & 0,092 \\
\hline 2013 & 0,047 & 0,103 & 0,023 & 0,271 & 0,329 & 0,231 & 0,083 \\
\hline 2014 & 0,041 & 0,097 & 0,016 & 0,291 & 0,365 & 0,208 & 0,081 \\
\hline
\end{tabular}

Nota: Fuente: Elaborado a partir de los microdatos de la EPH. 


\section{CUADRO A3}

INDICE DE KAITZ. ASALARIADOS DE LAS MGEYSP, 1995-2014

\begin{tabular}{|c|c|c|c|c|c|c|c|}
\hline \multirow{3}{*}{ Años } & \multicolumn{6}{|c|}{ Asalariados } & \multirow{3}{*}{ Todos } \\
\hline & \multicolumn{3}{|c|}{ Formales } & \multicolumn{3}{|c|}{ Informales } & \\
\hline & Todos & Con déficit & Sin déficit & Todos & Con déficit & Sin déficit & \\
\hline 1995 & 0,236 & 0,276 & 0,207 & 0,299 & 0,332 & 0,225 & 0,254 \\
\hline 1996 & 0,240 & 0,281 & 0,211 & 0,354 & 0,382 & 0,286 & 0,267 \\
\hline 1997 & 0,240 & 0,276 & 0,211 & 0,320 & 0,362 & 0,223 & 0,264 \\
\hline 1998 & 0,228 & 0,272 & 0,195 & 0,345 & 0,381 & 0,252 & 0,261 \\
\hline 1999 & 0,228 & 0,272 & 0,196 & 0,355 & 0,380 & 0,291 & 0,264 \\
\hline 2000 & 0,229 & 0,272 & 0,199 & 0,370 & 0,404 & 0,284 & 0,266 \\
\hline 2001 & 0,233 & 0,269 & 0,205 & 0,361 & 0,407 & 0,258 & 0,271 \\
\hline 2002 & 0,231 & 0,275 & 0,198 & 0,384 & 0,412 & 0,302 & 0,273 \\
\hline 2003 & 0,220 & 0,260 & 0,192 & 0,400 & 0,440 & 0,284 & 0,276 \\
\hline 2004 & 0,336 & 0,417 & 0,288 & 0,591 & 0,655 & 0,421 & 0,417 \\
\hline 2005 & 0,432 & 0,524 & 0,379 & 0,757 & 0,821 & 0,548 & 0,536 \\
\hline 2006 & 0,422 & 0,526 & 0,366 & 0,762 & 0,871 & 0,531 & 0,522 \\
\hline 2007 & 0,440 & 0,550 & 0,386 & 0,795 & 0,890 & 0,623 & 0,537 \\
\hline 2008 & 0,426 & 0,549 & 0,370 & 0,748 & 0,861 & 0,574 & 0,509 \\
\hline 2009 & 0,440 & 0,551 & 0,389 & 0,767 & 0,832 & 0,635 & 0,525 \\
\hline 2010 & 0,437 & 0,556 & 0,382 & 0,745 & 0,838 & 0,620 & 0,518 \\
\hline 2011 & 0,417 & 0,547 & 0,363 & 0,736 & 0,834 & 0,612 & 0,496 \\
\hline 2012 & 0,410 & 0,517 & 0,366 & 0,700 & 0,770 & 0,580 & 0,482 \\
\hline 2013 & 0,426 & 0,538 & 0,380 & 0,626 & 0,649 & 0,591 & 0,485 \\
\hline 2014 & 0,394 & 0,506 & 0,351 & 0,658 & 0,723 & 0,562 & 0,459 \\
\hline
\end{tabular}

Fuente: Elaborado a partir de los microdatos de la EPH.-INDEC. 


\section{CUADRO A4}

TASA DE INCUMPLIMIENTO DEL SM E INDICE DE KAITZ POR AREA URBANA

\begin{tabular}{|l|c|c|}
\hline Aglomerados & Tasa de incumplimiento & Kaitz \\
\hline Usuahia & 0,062 & 0,290 \\
Ciudad de Bs. As. & 0,092 & 0,344 \\
Río Gallegos & 0,099 & 0,516 \\
Comodoro Rivadavia & 0,140 & 0,340 \\
La Plata & 0,176 & 0,450 \\
Neuquén & 0,186 & 0,449 \\
Mar del Plata & 0,207 & 0,522 \\
Santa Rosa & 0,242 & 0,494 \\
Part. del GBA & 0,250 & 0,577 \\
Bahía Blanca & 0,267 & 0,625 \\
Córdoba & 0,289 & 0,564 \\
Río Cuarto & 0,293 & 0,604 \\
Mendoza & 0,294 & 0,640 \\
Rosario & 0,298 & 0,530 \\
La Rioja & 0,321 & 0,680 \\
Paraná & 0,332 & 0,672 \\
San Luis & 0,344 & 0,744 \\
Santa Fe & 0,347 & 0,634 \\
Posadas & 0,387 & 0,688 \\
Catamarca & 0,402 & 0,762 \\
Sant. del Estero & 0,426 & 0,781 \\
San Juan & 0,429 & 0,834 \\
Salta & 0,452 & 0,743 \\
Tucumán & 0,453 & 0,723 \\
Concordia & 0,459 & 0,861 \\
Corrientes & 0,474 & 0,814 \\
San Salvador de Jujuy & 0,991 \\
Resistencia & 0,500 & 0,954 \\
Formosa & 0,531 & 0,848 \\
\hline & 0,536 & \\
\hline
\end{tabular}

Fuente: Elaborado a partir de los microdatos de la EPH.-INDEC. 